\title{
Clinical Applicability of Adult Human Mesenchymal Stem Cell Therapy in the Treatment of Knee Osteoarthritis
}

This article was published in the following Dove Press journal: Stem Cells and Cloning: Advances and Applications

\section{Noor Buzaboon \\ Sfoug Alshammary}

Department of Molecular Medicine, College of Medicine and Medical Sciences, Princess Al-Jawhara Center for Molecular Medicine and Inherited Disorders, Arabian Gulf University, Manama, Bahrain
Correspondence: Sfoug Alshammary Email Sfougfhs@agu.edu.bh
Introduction: Osteoarthritis causes a progressive deterioration to the protective cartilage between the joints leading to chronic pain and disability. This review focuses on the intrinsic potential of MSCs to stabilize and repair the cartilage tissue of the knee joint in knee osteoarthritis (KOA) patients.

Methods: An online search through the PubMed database was conducted, limiting the search to the English language and human clinical trials within the past 5 years. Twentyone clinical trials passed the inclusion criteria. Combined, those trials involved the participation of 589 patients where the progress of the treatments was monitored between a 4-month to 7-years period. The cartilage volume and defects were observed through an MRI to provide an objective assessment. While the pain and knee function were monitored using KOOS, VAS, and WOMAC scoring scales providing a subjective assessment.

Results: MRI scans obtained from clinical trials demonstrate a slowed progression of cartilage degeneration and early signs of cartilage regeneration in KOA patients at the 12month follow-up period. No major adverse effects were observed post-intervention. The overall KOOS, WOMAC, and VAS scores in patients receiving MSC treatment were reduced, suggesting subjective improvements in knee function and pain reduction when compared to patients in the placebo group.

Conclusion: The use of MSC therapy is a valid form of treatment for KOA as it targets the disease itself rather than the symptoms. We found MSC therapy in KOA patients to be safe, effective, and feasible in its execution.

Keywords: adipose-derived mesenchymal stem cells, AD-MSC, bone-marrow mesenchymal stem cells, BM-MSC, umbilical-cord derived mesenchymal stem cells, UC-MSC

\section{Introduction}

Osteoarthritis (OA) is a biomechanical degenerative-joint disease. OA is characterized by the degradation of the articular cartilage, synovitis, subchondral bone lesions, osteophytes, and the hypertrophy of the joint capsule. ${ }^{1}$ Worldwide, it is approximated that $10 \%$ of men and $18 \%$ of women above the age of 65 years have symptomatic OA. ${ }^{2}$ A population-based cohort study conducted in 2008 showed that the lifetime risk of symptomatic KOA was $45 \%{ }^{3}$ In the United States, symptomatic KOA affects over 9 million adults over the age of 45 years. ${ }^{4}$ The high prevalence of this disease suggests that KOA will continue to have a major economic impact on healthcare systems. The number of individuals affected with KOA is likely to increase due to an ageing population, lack of exercise, and an ongoing obesity 
epidemic. The chronic pain caused by the progression of KOA affects the mobility of the patient eventually leading to the patient's disability. ${ }^{1,4}$

\section{Pathophysiology of Knee Osteoarthritis}

$\mathrm{KOA}$ is no longer thought of as an age-related degenerative disorder but is attributed to a multifactorial etiology; an interplay between various systemic and local factors.

Systemic risk factors include age, gender, race/ethnicity, genetics, congenital development, and diet. Females, above the age of 60 years are more likely to present with symptomatic KOA at $47 \%$ versus their male counterparts at $40 \%$. This is likely due to a hormonal imbalance observed during menopause. The risk of developing symptomatic KOA further increases by $60 \%$ when the patient has a body mass index of 30 or higher. $^{6}$

Local factors include obesity, injuries, occupational, and physical $/$ mechanical factors. ${ }^{6}$ It is estimated that $12 \%$ of $\mathrm{OA}$ arises secondary to post-traumatic injuries; such as the rupture of the Anterior cruciate ligament (ACL) where $80 \%$ of the patients show radiographic KOA within 14 years following the repair of the injury. ${ }^{7}$

These factors, along with the disruption of cytokine homeostasis (catabolic, anabolic, and regulatory pathways), result in biologic changes at the knee joint between the subchondral bone and the articular cartilage., 3 Biologic changes result in an increase in bone volume and trabecular thickness of the joints which are early signs observed in OA. As a result; the bone becomes stiffer with a reduced ability to absorb impact loads, thus increasing stress on the cartilage. Increased stress on the cartilage results in chondrocyte cell senescence. ${ }^{3}$ Degeneration of the articular cartilage is a pathologic hallmark of OA resulting in bony remodeling, osteophytes, capsular stretching, and weakness of peri-articular muscles. Sometimes, bone marrow lesions and synovitis can also be observed. ${ }^{3}$

The cartilage is a very complex tissue as it houses a diverse environment made up of chondrocytes, type II collagen, aggrecan (predominant proteoglycan), and an extensive water-based extracellular matrix. The extracellular matrix (ECM) contains a highly complicated network of cytokines and growth factors that are secreted by the synovial lining cells and chondrocytes. ${ }^{3}$

Disruption to the homeostasis in the synovial fluid triggered due to the prolonged presence of biologic stressors such as reactive oxygen species (ROS) and nitric oxide (NO) activates macrophages and NF- $\mathrm{k} \beta$ (nuclear factor kappa-light-chain-enhancer of activated $\mathrm{B}$ cells). NF- $\mathrm{k} \beta$ plays a critical role in immune regulation and expression of cytokines involved in inflammation. NF$k \beta$ induces the release of proinflammatory cytokines such as interleukin-1 $\beta$ (IL-1 $\beta$ ) and Tumor Necrosis Factor $\alpha$ $(\mathrm{TNF} \alpha)^{8}$

The physiological hallmark of OA is characterized by an increase in inflammatory cytokine expression (IL-1 $\beta$ and TNF- $\alpha$ ) which further induces the production of other pro-inflammatory factors (such as IL-8, IL-6, matrix metalloproteinases, leukotriene inhibiting factor, proteases, and prostaglandin E2) causing an inflammation within the synovial fluid. ${ }^{5,9}$ The inflammation process is exacerbated when IL- 6 and IL- $1 \alpha$ induce the release of Interferon- $\gamma$ (INF- $\gamma$ ) which promotes CD4+ T regulatory cells production and elevated levels of Tumor Growth Factors- $\beta$ (TGF $\beta)$. The result of this is heightened inflammation process is directly associated with osteophyte formation in the lateral tibiofemoral joint and the medial patellofemoral joint. ${ }^{8}$

Proteinases such as MMP-9 and MMP-13 are overexpressed by inflammatory cytokines which target collagen and aggrecan for degradation. Aggrecan is the predominant form of proteoglycan (PG) found in the cartilage. Its degradation is associated with the upregulation of MMPs and ADAMTs (especially ADAMT-5 and ADAMT-4). ${ }^{8}$ The congregation of all these factors around the joint leads to a pro-catabolic state, the development of $\mathrm{OA}$, and gradual degradation of the matrix. ${ }^{5,8,9}$

Lipid peroxidation is another factor known to cause the degradation of the ECM and the cartilage. Lipid peroxidation simply refers to the oxidation of polyunsaturated fatty acids and the production of aldehydes and hydroperoxides, which are known to cause cartilage degradation. ${ }^{8}$

\section{Conventional Treatment}

$\mathrm{KOA}$ is commonly diagnosed using a radiographic reference standard; the Kellgren-Lawrence $(\mathrm{K} / \mathrm{L})$ grading scheme, which is an overall joint scoring system. The $\mathrm{K} / \mathrm{L}$ grading scheme of $\mathrm{OA}$ measures five levels from 0 to 4 .

Current medical treatments for KOA are focused on symptomatic control instead of disease modification.

For $\mathrm{K} / \mathrm{L}$ Grade $0-1$, care is usually limited to occasional analgesics such as acetaminophen, NSAIDs, or opioids. Patients are also encouraged to exercise and 
strengthen their quadriceps muscles, attend physical therapy, and in some cases, weight reduction is advised. Some patients are also advised to wear a valgus-directing force brace. $^{6}$

As KOA progresses to Grade $\geq 2$, Intra-articular (IA) corticosteroid injections and analgesics are commonly used for symptomatic care. $\mathrm{K} / \mathrm{L}$ Grade $\geq 2$ is considered an osteophyte with more severe grades demonstrating joint space narrowing, cysts, sclerosis, and deformity of the joint. ${ }^{6}$

After exhausting the non-surgical treatment options, uni-compartmental, or total knee arthroplasty (TKA) at Grade 3-4 become a viable option for patients. TKA procedure is not only invasive and costly but also comes with a risk for significant complications. It is estimated that $20 \%$ of patients will continue to face persistent pain (maybe even need a revision TKA) or lose function within 12 months of the procedure. ${ }^{5,8}$ TKA procedure for symptomatic KOA patients directly accounts for $61 \%$ of the medical costs used in OA-related cost of care. ${ }^{6}$

\section{The Intrinsic Potential of Stem Cell Therapies}

Stem cells are unspecialized multipotent cells that can differentiate into various cell types and renew themselves indefinitely. These cells are readily isolated from certain protected niches within the body, such as the bone marrow, adipose tissue, and synovium. ${ }^{10}$ There are three types of stem cells; Embryonic Stem Cells (ESCs), Adult Stem Cells (ASCs), and induced Progenitor Stem Cells (iPSCs).

Mesenchymal Stem Cells (MSCs) are ASCs that originate from the mesoderm and can differentiate into various connective tissue cells such as chondrocytes, adipocytes, and osteocytes. ${ }^{7}$

MSCs can be immunophenotypically identified using flow cytometry and tested by anti-HLA-panel reactive antibodies (PRA) against class I and II HLAs. MSCs show mesenchymal surface markers for CD90, CD44, CD73, and CD105, and the absence of hematopoietic cell surface markers for CD34, CD19, CD14, and CD45. 1,10

Articular cartilage tissue has a slow and limited regenerative capacity. ${ }^{11}$ Resident MSCs are considered repair agents that are normally found in low numbers around the superficial layer of the cartilage. In KOA, the normal function of resident MSCs is disrupted, leading to the inflammation of the synovial membrane due to the infiltration of CD4+ T-cells and CD68 macrophages. Resident MSCs have been observed to suppress synovial activation and indirectly exacerbate cartilage damage. ${ }^{9}$

Non-resident MSCs have an inherent tendency to differentiate into chondrocytes in-vitro and induce the proliferation of resident progenitor cells in-vivo. Although the exact mechanism of MSCs on pain reduction and cartilage repair is not fully understood, MSCs create a repair microenvironment via paracrine signalling and the recruitment of local endogenous cells. The repair microenvironment is created by inducing chondroprogenitors, growth factors, and extracellular matrix proteins (such as aggrecan and type II collagen), to halt disease progression. ${ }^{12}$ The presence of these factors in the affected tissue area causes the inhibition of pro-inflammatory cytokines, neutrophils, and the cytotoxic activity of natural killer (NK) cells. The proliferation of T-cells is suppressed. The maturation of monocytes is inhibited while the growth of regulatory T-cells is promoted. ${ }^{13}$ This process leads to the up-regulation of senescent metabolically active chondrocytes, initiating repair of the damaged tissue.

Research suggests that engrafted MSCs in the joint are activated to express the Indian hedgehog and other 'hit and run' genes. These genes promote the collagen II expression, the secretion of a wide range of anti-inflammatory cytokines and analgesic peptides. ${ }^{14,15}$ Clinical trials, using MSCs have shown the in-vivo capacity of MSCs to survive, thrive, and initiate repair via neo-cartilage formation when implanted in the articular milieu. ${ }^{11,15}$

These disease-modifying qualities make MSC therapy an invaluable tool for $\mathrm{OA}$ disease stabilization and modification. . $^{5,15}$

\section{MSC Interventions to Treat KOA}

The objective behind employing stem cell-based therapies is the potential for developing a cost-effective, novel treatment from an abundant cell source, with "hypoimmunogenic" benefits, while being minimally invasive.

MSC therapies are delivered to KOA patients using three main intervention pathways: tissue-engineered MSC transplants (MSC gel scaffolds), Intra-articular (IA) Injections (scaffold-free or combined w/fibrin gel, HA or PRP), and cell-free injection of exosome into the damaged articular joint. IA injections are used in most clinical trials, as they are less invasive and demonstrate ease in delivering MSCs when compared to arthroscopic MSC gelscaffold transplants. The use of IA injection is more suitable in the treatment of mild-to-moderate severity of 
KOA, whereas MSC transplants are most commonly used in the treatment of severe KOA. MSC transplants are usually implanted into the knee joint following an arthroscopic or surgical procedure. ${ }^{10}$

MSC exosomes are extracellular vesicles formed by the inward budding of the endosomal membranes that have a range in diameter of $30-150 \mathrm{~nm}$. MSC exosomes can be derived from the same tissue sources that are used to obtain MSCs (bone-marrow, adipose, umbilical cord, and placenta). These exosomes carry a complex cargo of nucleic acids, proteins, and lipids that mediate tissue repair in the damaged knee cartilage. Recent trials have reported the use of cell-free, MSC-based fractions, also known as MSC exosomes to develop a treatment that is readily available with improved efficacy. ${ }^{10}$

\section{Methods}

A computer-based online search through PubMed database was conducted on March 27, 2020. Published articles that met the inclusion criteria were selected for critical review and analysis. The method section discusses the eligibility of the clinical trials, search filters that were used in the selection process, invalid/missing data from the clinical trials, data collection, and analysis.

Research Question: Is MSC therapy an efficacious form of treatment for KOA?

\section{Eligibility of the Clinical Trial Inclusion Criteria}

The articles selected for the review from the database were limited to the English language and published between March 2015 and March 2020. The clinical trials selected focused on the treatment of KOA on human subjects using Adult human Mesenchymal Stem cells only. The articles included disregarded the origin of the MSCs (adipose/bone marrow/placental/umbilical derived), phase of the clinical trial, the population size, age of the patients, and the method of intervention delivery.

\section{Exclusion Criteria}

The clinical trials that were eliminated from this review included animal only models, other drug therapies (cellfree formulations), iPSCs (induced pluripotent stem cells), and embryonic stem cells. Trials were excluded, if they were older than 5 years, did not exclusively assess the clinical efficacy of the treatment on the intra-articular knee joint and the availability of full-text formats.

\section{Search Filters}

The phrase below was used to search for "knee osteoarthritis \& mesenchymal stem cell therapy" through the PubMed database on March 27, 2020.

((“osteoarthritis, knee"[MeSH Terms] OR ("osteoarthritis"[All Fields] AND "knee"[All Fields]) AND ("mesenchymal stem cells"[MeSH Terms] OR ("mesenchymal"[All Fields] AND "stem"[All Fields] AND “cells"[All Fields]) AND ("therapy"[Subheading] OR "therapy"[All Fields] OR "treatment"[All Fields] OR "therapeutics"[MeSH Terms] AND (Clinical Trial[ptyp] AND “loattrfull text"[sb] AND “2015/03/30"[PDat]: “2020/03/27”[PDat] AND “humans”[MeSHTerms]).

\section{Data Collection}

The data collected for this review can be split into two major sections; a table that qualitatively summarizes all the clinical trials being reviewed, and a quantitative analysis that uses Microsoft Excel software to process the data.

The qualitative analysis accounted for general information collected from each clinical trial such as; the number of patients enrolled, age group, the clinical trial, the intervention, results, outcomes, and the limitation of each trial. Displaying data in this format is critical as it lays a foundation on which quantitative analysis can build upon.

Table 1, in the Results section, compares and summarizes the clinical trials being studied in this review.

The clinical trials being reviewed measure many variables, so a simple analysis displaying a definite conclusion in one graph or table is unlikely. Therefore, the data compiled from the clinical trials were arranged categorically into separate tables, enabling analysis of the same variables. The data retrieved from the tables were further analyzed into graphs, visually explaining the results achieved from the experiments.

\section{Invalid/Missing Data}

The clinical trials assessed in this review were methodically heterogeneous in their experimental design (patient age, cell source, dose, frequency of intervention, and cell processing methods). This makes it difficult to compare results from various trials. Some studies lack blinding, some lack placebo groups, and some studies measure different variables (VAS instead of KOOS, X-ray of cartilage instead of measuring the cartilage density through MRI).

\section{Data Analysis}

The data is categorized into three sections: 
Table I Clinical Trials Selected That Utilize Adult Human MSCs in the Treatment of KOA

\begin{tabular}{|c|c|c|c|c|c|c|}
\hline Study & $\begin{array}{l}\text { Patients } \\
\text { Enrolled }\end{array}$ & Method & Intervention & Results & Conclusion & Limitation \\
\hline $\begin{array}{l}\text { Freitag } \\
\text { J. et al, } 2019^{5}\end{array}$ & 30 (Age I8+) & $\begin{array}{l}\text { Randomized } \\
\text { controlled } \\
\text { trial (RCT) }\end{array}$ & $\begin{array}{l}\text { Intra-articular } \\
\text { injections of } \\
\text { autologous ADMSCs: } \\
\text { Placebo vs single } \\
\text { (baseline) vs double } \\
\text { dose ( } 100 \times 10^{6} \\
\text { ADMSCs) at baseline } \\
\text { and } 6 \text { months. }\end{array}$ & $\begin{array}{l}\text { Significant reduction in } \\
\text { pain and functional } \\
\text { improvement of the knee } \\
\text { joint at I } 2 \text { months. MRI } \\
\text { and KOOS indicated } \\
\text { disease modification. } \\
\text { Double-dose treatment } \\
\text { achieved more consistent } \\
\text { OA stabilization. }\end{array}$ & $\begin{array}{l}\text { Safe and effective } \\
\text { therapy of KOA. No } \\
\text { adverse AE observed. }\end{array}$ & $\begin{array}{l}\text { First published RCT } \\
\text { studying the effect of } \\
\text { autologous expanded } \\
\text { ADMSCs on KOA. } \\
\text { Additional research } \\
\text { with direct } \\
\text { comparison required. }\end{array}$ \\
\hline $\begin{array}{l}\text { Frietag } \\
\text { J. et al, } 2015^{10}\end{array}$ & 40 (Age I8-50) & $\begin{array}{l}\text { Pilot, single- } \\
\text { center RCT }\end{array}$ & $\begin{array}{l}\text { Placebo vs } \\
\text { Arthroscopic M.F. vs } \\
\text { Arthroscopic M.F. w/ } \\
\text { intra- articular } \\
\text { injection of } \\
\text { autologous ADMSCs. }\end{array}$ & $\begin{array}{l}\text { The primary outcome of } \\
\text { MRI and KOOS is being } \\
\text { assessed with I2-month } \\
\text { follow-up. Hypothesis: } \\
\text { Treatment w/ADMSCs } \\
\text { may result in increased } \\
\text { type II collagen } \\
\text { deposition and } \\
\text { regeneration of hyaline- } \\
\text { like cartilage. AEs will be } \\
\text { noted. }\end{array}$ & $\begin{array}{l}\text { The trial was in } \\
\text { progress. }\end{array}$ & $\begin{array}{l}\text { Lack of blinding in the } \\
\text { trial, insufficient data } \\
\text { for calculations due to } \\
\text { small sample size and } \\
\text { a larger phase-III RCT } \\
\text { requiring greater } \\
\text { funding. }\end{array}$ \\
\hline $\begin{array}{l}\text { Lamo- } \\
\text { Espinosa } \\
\text { J. et al, } 2016^{22}\end{array}$ & 30 (Age 50-80) & $\begin{array}{l}\text { Multicentre } \\
\text { RCT }\end{array}$ & $\begin{array}{l}\text { Intra-articular } \\
\text { injections of } \\
\text { autologous BM-MSCs: } \\
\text { Placebo (H.A. only) vs } \\
\text { Low dose (H.A. w/10 } \\
\times 10^{6} \text { BM-MSCs) vs } \\
\text { High dose (H.A. w/ } \\
100 \times 10^{6} \text { BM-MSCs). }\end{array}$ & $\begin{array}{l}\text { Improvement in VAS \& } \\
\text { WOMAC scores at I2- } \\
\text { months for both } \\
\text { treatment groups. MRI } \\
\text { demonstrates a decrease } \\
\text { in joint damage high-dose } \\
\text { treatment group. X-ray } \\
\text { revealed decrease in joint } \\
\text { space width only in } \\
\text { control group. }\end{array}$ & $\begin{array}{l}\text { Treatment of KOA w/ } \\
\text { H.A. and B.M.- MSCs } \\
\text { is safe and feasible, } \\
\text { especially in the high- } \\
\text { dose treatment } \\
\text { group. }\end{array}$ & $\begin{array}{l}\text { Ethical issues } \\
\text { prevented a double- } \\
\text { blinded trial. }\end{array}$ \\
\hline $\begin{array}{l}\text { Lamo- } \\
\text { Espinosa } \\
\text { J. et al, } 2018^{23}\end{array}$ & $\begin{array}{l}\text { Same Patients } \\
\text { as Lamo- } \\
\text { Espinosa J., et al } \\
(2016) ; \\
3 \text { patients } \\
\text { opted-out of } \\
\text { long-term } \\
\text { follow up }\end{array}$ & $\begin{array}{l}\text { Multi- center } \\
\text { RCT }\end{array}$ & $\begin{array}{l}\text { Long-term follow up of } \\
4 \text { years with no } \\
\text { additional } \\
\text { intervention. } \\
\text { Studying the clinical } \\
\text { and radiographic } \\
\text { evolution of KOA } \\
\text { patients as a follow up } \\
\text { to HA+ BM- MSCs } \\
\text { treatment. }\end{array}$ & $\begin{array}{l}\text { Positive results in both } \\
\text { VAS and WOMAC } \\
\text { scores for low and high } \\
\text { dose groups. No clinical } \\
\text { significance observed } \\
\text { between the low and } \\
\text { high dose treatment } \\
\text { groups. }\end{array}$ & $\begin{array}{l}\text { Clinical and } \\
\text { functional } \\
\text { improvement of the } \\
\text { knee joint when } \\
\text { compared to the } \\
\text { control group. }\end{array}$ & $\begin{array}{l}\text { Further research } \\
\text { leading to phase-III } \\
\text { required to better } \\
\text { understand the } \\
\text { efficacy of BM-MSCs } \\
\text { in treating KOA. }\end{array}$ \\
\hline $\begin{array}{l}\text { Al Najar } \\
\text { M. et } \mathrm{al}^{24}\end{array}$ & $\begin{array}{l}\text { I3 (Mean age } 50 \\
\text { and under } 65 \\
\text { years old) }\end{array}$ & $\begin{array}{l}\text { Prospective } \\
\text { Phase I open- } \\
\text { label clinical } \\
\text { trial }\end{array}$ & $\begin{array}{l}\text { Two doses of Intra- } \\
\text { articular injections of } \\
\text { autologous BM-MSCs } \\
\text { I month apart } \\
\text { (totaling } 61 \times 10^{6} \\
\pm 0.6 \times 10^{6} \text { ). }\end{array}$ & $\begin{array}{l}\text { Improvement in cartilage } \\
\text { thickness and KOOS } \\
\text { scores observed. No } \\
\text { lasting AEs observed } \\
\text { within 24-month from } \\
\text { treatment. }\end{array}$ & $\begin{array}{l}\text { Safe drug } \\
\text { administration, } \\
\text { clinical cartilage } \\
\text { thickness and } \\
\text { functional } \\
\text { improvement of the } \\
\text { knee joint. }\end{array}$ & $\begin{array}{l}\text { No control group and } \\
\text { a limited number of } \\
\text { patients enrolled. } \\
\text { Larger sample size } \\
\text { required to better } \\
\text { understand the } \\
\text { efficacy of BM-MSCs } \\
\text { therapy in KOA. }\end{array}$ \\
\hline
\end{tabular}

(Continued) 
Table I (Continued).

\begin{tabular}{|c|c|c|c|c|c|c|}
\hline Study & $\begin{array}{l}\text { Patients } \\
\text { Enrolled }\end{array}$ & Method & Intervention & Results & Conclusion & Limitation \\
\hline $\begin{array}{l}\text { Shetty } \\
\text { A. et } \mathrm{al}^{25}\end{array}$ & 60 (Age 19-61) & $\begin{array}{l}\text { Prospective } \\
\text { phase I open- } \\
\text { label clinical } \\
\text { trial }\end{array}$ & $\begin{array}{l}\text { MCIC- Arthroscopic } \\
\text { procedure utilizing } \\
\text { CO2 insufflation. }\end{array}$ & $\begin{array}{l}\text { Postoperative clinical } \\
\text { assessments } \\
\text { demonstrated significant } \\
\text { improvement of } P<0.05 \\
\text { in KOOS, LKSS and } \\
\text { IKDC. }\end{array}$ & $\begin{array}{l}\text { Regeneration of the } \\
\text { cartilage observed. } \\
\text { Effective and single- } \\
\text { stage treatment. No } \\
\text { serious AE recorded } \\
\text { from operative } \\
\text { procedures. }\end{array}$ & $\begin{array}{l}\text { Not enough studies } \\
\text { supporting long-term } \\
\text { effects and insufficient } \\
\text { health economic } \\
\text { justification of the } \\
\text { procedure. No } \\
\text { placebo groups. }\end{array}$ \\
\hline Wang Y. et $\mathrm{al}^{7}$ & 17 (Age 18-40) & $\begin{array}{l}\text { Double- blind } \\
\text { RCT }\end{array}$ & $\begin{array}{l}\text { Intra-articular } \\
\text { injections: Placebo }(\mathrm{H} . \\
\text { A. only) group vs } \\
\text { allogenic MPC+HA } \\
\left(75 \times 10^{6}\right) \text { group. }\end{array}$ & $\begin{array}{l}\text { Moderate arthralgia and } \\
\text { swelling in } 4 \text { patients. MPC } \\
+ \text { HA patients } \\
\text { demonstrated } \\
\text { improvement in KOOS, } \\
\text { ADL and SF- } 36 \text { score } \\
(P<0.05) \text { compared to the } \\
\text { placebo group. }\end{array}$ & $\begin{array}{l}\text { Treatment was safe } \\
\text { and well tolerated. } \\
\text { Improvement in } \\
\text { clinical and structural } \\
\text { outcomes. }\end{array}$ & $\begin{array}{l}\text { Further investigations } \\
\text { required to modulate } \\
\text { the understanding of } \\
\text { the pathological } \\
\text { processes responsible } \\
\text { for regenerative } \\
\text { restoration of } \\
\text { articular cartilage. }\end{array}$ \\
\hline $\begin{array}{l}\text { Gupta P. } \\
\text { K. et al }{ }^{15}\end{array}$ & 60 (Age 40-70) & $\begin{array}{l}\text { Multi- center, } \\
\text { double- blind } \\
\text { RCT }\end{array}$ & $\begin{array}{l}\text { Intra-articular } \\
\text { injections: Placebo }(\mathrm{H} \text {. } \\
\text { A. only) vs different } \\
\text { doses of Stempeucel } \\
(25,50,75 \text { or } 150 \\
\times 10^{6} \text { cells) followed } \\
\text { by } 2 \mathrm{~mL} \text { H.A. }\end{array}$ & $\begin{array}{l}\text { Stempeucel } \\
\text { administration resulted } \\
\text { in improvements in } \\
\text { qualitative parameters } \\
\text { (VAS, ICOAP \& } \\
\text { WOMAC) in all } \\
\text { treatment groups } \\
\text { compared to placebo at } \\
\text { I2 months follow- up. } \\
\text { Higher dose groups } \\
\text { demonstrated mild- } \\
\text { moderate AEs. }\end{array}$ & $\begin{array}{l}75 \times 10^{6} \text { MSC } \\
\text { treatment group } \\
\text { demonstrated most } \\
\text { effective } \\
\text { improvement in } \\
\text { clinical outcome. }\end{array}$ & $\begin{array}{l}\text { Larger sample size } \\
\text { required to } \\
\text { demonstrate the } \\
\text { therapeutic efficacy of } \\
\text { Stempeucel in the } \\
\text { treatment of KOA. }\end{array}$ \\
\hline $\begin{array}{l}\text { Pers } Y \text {. } \\
\text { M. et } \mathrm{al}^{26}\end{array}$ & I8 (Age 50-75) & $\begin{array}{l}\text { Bicentric, } \\
\text { uncontrolled, } \\
\text { open phase } \\
\text { I trial }\end{array}$ & $\begin{array}{l}\text { Intra-articular } \\
\text { injections of ASCs: } \\
\text { low dose }\left(2 \times 10^{6}\right) \text {, } \\
\text { medium dose } \\
\left(10 \times 10^{6}\right) \text {, and high } \\
\text { dose }\left(50 \times 10^{6}\right) \text {. }\end{array}$ & $\begin{array}{l}\text { Clinical improvement and } \\
\text { reduction in pain levels } \\
\text { were recorded for all } \\
\text { treatment groups. } \\
\text { Statistically } \\
\text { significant improvement } \\
\text { was only seen in the low- } \\
\text { dose treatment group. }\end{array}$ & $\begin{array}{l}\text { Study demonstrated } \\
\text { safety, drug } \\
\text { tolerability and } \\
\text { efficacy of the } \\
\text { treatment. }\end{array}$ & $\begin{array}{l}\text { Short-term study (6 } \\
\text { months), } \\
\text { uncontrolled } \\
\text { experiment and small } \\
\text { sample size. }\end{array}$ \\
\hline $\begin{array}{l}\text { Davatchi } \\
\text { F. et } \text { al }^{9}\end{array}$ & 4 (Age 55-65) & $\begin{array}{l}\text { Long-term } \\
\text { follow up (5 } \\
\text { years) of } \\
\text { a 6-month } \\
\text { preliminary } \\
\text { study }\end{array}$ & $\begin{array}{l}\text { Transplant of MSCs in } \\
\text { the worse knee only } \\
\left(8 / 9 \times 10^{6} \mathrm{MSCs}\right)\end{array}$ & $\begin{array}{l}\text { Improved VAS, } \\
\text { functional outcome and } \\
\text { reduced pain scores } \\
\text { (PGA) for treated knee } \\
\text { compared to untreated } \\
\text { knee. }\end{array}$ & $\begin{array}{l}\text { Early intervention } \\
\text { utilizing MSCs would } \\
\text { demonstrate } \\
\text { promising long-term } \\
\text { results. }\end{array}$ & $\begin{array}{l}\text { Future studies are } \\
\text { required with a larger } \\
\text { population. }\end{array}$ \\
\hline Song $\mathrm{Y}$. et al' & I8 (Age 40-70) & $\begin{array}{l}\text { Prospective } \\
\text { uncontrolled } \\
\text { clinical trial }\end{array}$ & $\begin{array}{l}\text { Three Intra- articular } \\
\text { injections with } \\
\text { ADMSCs: low-dose } \\
\left(1 \times 10^{7}\right) \text {, mid-dose } \\
\left(2 \times 10^{7}\right) \text { and high-dose } \\
\left(5 \times 10^{7}\right) \text { for } 96 \text { weeks. }\end{array}$ & $\begin{array}{l}\text { Improved pain, function } \\
\text { and cartilage volume at } \\
\text { the knee joint. The high- } \\
\text { dose group exhibited the } \\
\text { highest improvement. }\end{array}$ & $\begin{array}{l}\text { ADMSCs treatment } \\
\text { demonstrated safety } \\
\text { and improvement of } \\
\text { function at the knee } \\
\text { joint. }\end{array}$ & $\begin{array}{l}\text { Lack of placebo/ } \\
\text { control group and } \\
\text { small sample size. }\end{array}$ \\
\hline
\end{tabular}

(Continued) 
Table I (Continued).

\begin{tabular}{|c|c|c|c|c|c|c|}
\hline Study & $\begin{array}{l}\text { Patients } \\
\text { Enrolled }\end{array}$ & Method & Intervention & Results & Conclusion & Limitation \\
\hline $\begin{array}{l}\text { Hernigou } \\
\text { P. et } \mathrm{al}^{27}\end{array}$ & 30 (Age I8-4I) & $\begin{array}{l}\text { Prospective } \\
\text { RCT }\end{array}$ & $\begin{array}{l}\text { Bilateral KOA } \\
\text { secondary to ON; } \\
\text { One knee received } \\
\text { TKA \& the other } \\
\text { received bone } \\
\text { marrow graft } \\
\text { (6500MSCs } / \mathrm{mL}) \text { to } \\
\text { subchondral bone. }\end{array}$ & $\begin{array}{l}\text { Surgical complications } \\
\text { were more frequent after } \\
\text { TKA. Improvement } \\
\text { observed in cartilage and } \\
\text { bone marrow lesions at } \\
\text { the injection site of BM- } \\
\text { graft therapy on the other } \\
\text { knee. }\end{array}$ & $\begin{array}{l}\text { Treatment with BM- } \\
\text { graft was effective, } \\
\text { with fewer AEs and } \\
\text { faster recovery } \\
\text { compared to the } \\
\text { TKA procedure. }\end{array}$ & $\begin{array}{l}\text { Invasive surgical } \\
\text { procedure and long- } \\
\text { recovery time. }\end{array}$ \\
\hline Vega A. et al ${ }^{14}$ & 30 (Age 18-75) & $\begin{array}{l}\text { Multicenter } \\
\text { RCT }\end{array}$ & $\begin{array}{l}\text { Intra-articular } \\
\text { injections: Placebo } \\
\text { group (60mg HA } \\
\text { only) vs treatment } \\
\text { group (allogeneic BM- } \\
\text { MSCs } 40 \times 10^{6} \text { and } \\
\text { HA) followed for } 12 \\
\text { months. }\end{array}$ & $\begin{array}{l}\text { Treatment with allogeneic } \\
\text { MSCs demonstrated } \\
\text { safety, feasibility and } \\
\text { clinical efficacy. } \\
\text { T2 measurements } \\
\text { indicated an } \\
\text { improvement in cartilage } \\
\text { quality. }\end{array}$ & $\begin{array}{l}\text { Allogeneic BM-MSC } \\
\text { therapy is a valid } \\
\text { alternative to } \\
\text { autologous MSCs } \\
\text { because it is more } \\
\text { convenient and less } \\
\text { invasive treatment } \\
\text { alternative. }\end{array}$ & $\begin{array}{l}\text { Future studies utilizing } \\
\text { both allogenic and } \\
\text { autologous MSCs are } \\
\text { required to provide } \\
\text { a better comparison } \\
\text { between the efficacy of } \\
\text { each type of MSC. }\end{array}$ \\
\hline $\begin{array}{l}\text { Bastos } \\
\text { R. et } \mathrm{al}^{28}\end{array}$ & $\begin{array}{l}18 \text { (Age } 57.6 \pm \\
9.6 \text { years) }\end{array}$ & $\begin{array}{l}\text { Randomized } \\
\text { uncontrolled } \\
\text { trial }\end{array}$ & $\begin{array}{l}\text { Intra-articular } \\
\text { injections: autologous } \\
\text { MSCs only vs } \\
\text { autologous MSCs+ } \\
\text { PRP followed for } 12 \\
\text { months }\end{array}$ & $\begin{array}{l}\text { Both MSC \& PRP+MSC } \\
\text { indicated significant } \\
\text { improvements in pain, } \\
\text { function and activities of } \\
\text { daily living ( } P<0.05) \text {. } \\
\text { Minimal AEs observed. }\end{array}$ & $\begin{array}{l}\text { Adding PRP to the } \\
\text { treatment did not } \\
\text { indicate better clinical } \\
\text { efficacy of MSC } \\
\text { treatment. Both } \\
\text { treatments were safe, } \\
\text { minimally invasive and } \\
\text { tolerable. }\end{array}$ & $\begin{array}{l}\text { Lack of placebo/ } \\
\text { control group and } \\
\text { small sample size. }\end{array}$ \\
\hline Soler R. et al ${ }^{\prime \prime}$ & $\begin{array}{l}15 \\
(\text { Median age = } \\
52 \text { years })\end{array}$ & $\begin{array}{l}\text { Prospective } \\
\text { Phase I-II, } \\
\text { open-label, } \\
\text { single-dose, } \\
\text { single-arm } \\
\text { clinical trial }\end{array}$ & $\begin{array}{l}\text { Intra-articular } \\
\text { infusion: }\left(40.9 \times 10^{6}\right. \\
\left. \pm 0.4 \times 10^{6}\right) \\
\text { autologous BM-MSCs. }\end{array}$ & $\begin{array}{l}\text { Treatment decreased the } \\
\text { intensity of pain. Physical } \\
\text { improvement maintained } \\
\text { for } 12 \text { months post- } \\
\text { treatment. Mild AEs } \\
\text { were recorded. }\end{array}$ & $\begin{array}{l}\text { Treatment } \\
\text { demonstrated safety, } \\
\text { tolerability, cartilage } \\
\text { repair and } \\
\text { improvement of QoL } \\
\text { (long- term benefit } \\
\text { up to } 4 \text { years post- } \\
\text { intervention). }\end{array}$ & $\begin{array}{l}\text { Lack of placebo/ } \\
\text { control group and } \\
\text { small sample size. }\end{array}$ \\
\hline $\begin{array}{l}\text { Koh Y. } \\
\text { G. et } \text { al }^{29}\end{array}$ & 80 (Age 18-50) & $\begin{array}{l}\text { Unblinded } \\
\text { RCT }\end{array}$ & $\begin{array}{l}\text { Arthroscopies: } \\
\text { placebo (M.F. only) vs } \\
\text { treatment group } \\
\text { (ADSCs + M.F.) } \\
\text { followed and assessed } \\
\text { for } 24 \text { months. }\end{array}$ & $\begin{array}{l}\text { Improvement in KOOS } \\
\text { scores and cartilage } \\
\text { coverage in the } \\
\text { treatment group versus } \\
\text { placebo group. ADL } \\
\text { scores were not } \\
\text { significantly different } \\
\text { between both groups. }\end{array}$ & $\begin{array}{l}\text { Treatment group } \\
\text { demonstrated } \\
\text { improvement in pain, } \\
\text { function and radiologic } \\
\text { imaging. Significant } \\
\text { structural repair was } \\
\text { observed in the } \\
\text { cartilage of the knee in } \\
\text { the treatment group. }\end{array}$ & $\begin{array}{l}\text { Larger sample size } \\
\text { and long-term follow } \\
\text { up required to } \\
\text { demonstrate } \\
\text { therapeutic efficacy. }\end{array}$ \\
\hline $\begin{array}{l}\text { Emadedin } \\
\text { M. et al }^{12}\end{array}$ & $\begin{array}{l}43 \\
(\text { Median age = } \\
53 \text { years })\end{array}$ & $\begin{array}{l}\text { Single centre, } \\
\text { Phase } \mathrm{I} / \mathrm{ll} \text {, } \\
\text { Triple blind } \\
\text { RCT }\end{array}$ & $\begin{array}{l}\text { Intra-articular } \\
\text { implantation: Placebo } \\
\text { ( } 5 \mathrm{~mL} \text { Saline) vs } \\
\text { treatment group } \\
\left(40 \times 10^{6} \text { autologous }\right. \\
\text { BM-MSCs). }\end{array}$ & $\begin{array}{l}\text { The treatment group } \\
\text { demonstrated } \\
\text { improvements in the } \\
\text { total WOMAC score } \\
\text { over six months follow- } \\
\text { up. No major AEs } \\
\text { recorded. }\end{array}$ & $\begin{array}{l}\text { MSCs treatment } \\
\text { provided patients } \\
\text { with the significant } \\
\text { clinical improvement } \\
\text { compared to the } \\
\text { placebo group. }\end{array}$ & $\begin{array}{l}\text { Short-term study (6 } \\
\text { months) and larger } \\
\text { cohort required to } \\
\text { better assess the } \\
\text { efficacy of the } \\
\text { treatment. }\end{array}$ \\
\hline
\end{tabular}

(Continued) 
Table I (Continued).

\begin{tabular}{|c|c|c|c|c|c|c|}
\hline Study & $\begin{array}{l}\text { Patients } \\
\text { Enrolled }\end{array}$ & Method & Intervention & Results & Conclusion & Limitation \\
\hline $\begin{array}{l}\text { Shadmanfar } \\
\text { S. et } \text { al }^{13}\end{array}$ & $\begin{array}{l}30 \\
\text { (Age 18-65) }\end{array}$ & $\begin{array}{l}\text { Single center, } \\
\text { Phase I/II, } \\
\text { Triple blind } \\
\text { RCT- w/ } \\
\text { Rheumatoid } \\
\text { Arthritis (R. } \\
\text { A.) patients. }\end{array}$ & $\begin{array}{l}\text { Intra-articular } \\
\text { implantation: Placebo } \\
\text { (Saline) vs treatment } \\
\text { group ( } 40 \times 10^{6} \\
\text { autologous BM- } \\
\text { MSCs). }\end{array}$ & $\begin{array}{l}\text { Patients receiving } \\
\text { treatment demonstrated } \\
\text { improved WOMAC and } \\
\text { VAS scores during the } \\
\text { 12-month follow-up } \\
\text { compared to placebo. } \\
\text { Reductions to } \\
\text { methotrexate and } \\
\text { prednisone use, was } \\
\text { noticed in treatment } \\
\text { group. }\end{array}$ & $\begin{array}{l}\text { MSCs implantation } \\
\text { demonstrated safety, } \\
\text { tolerability, and } \\
\text { a positive trend in } \\
\text { clinical efficacy. }\end{array}$ & $\begin{array}{l}\text { Improvement could } \\
\text { not be significantly } \\
\text { sustained after } 12 \\
\text { months. }\end{array}$ \\
\hline $\begin{array}{l}\text { Mastas } \\
\text { J. et } \mathrm{al}^{30}\end{array}$ & $\begin{array}{l}26 \\
\text { (Age 40-65) }\end{array}$ & $\begin{array}{l}\text { Pilot Phase I/ } \\
\text { II, Double- } \\
\text { blind, RCT }\end{array}$ & $\begin{array}{l}\text { Intra-articular } \\
\text { injections: } \\
\text { Placebo (HA only at } \\
\text { baseline and 6-month) } \\
\text { vs Group-I (UC-MSCs } \\
\text { at baseline and HA at } \\
\text { 6-month) vs Group-2 } \\
\text { (UC-MSCs at baseline } \\
\text { and 6-month). } \\
\text { Treatment injections } \\
\text { contained: } 20 \times 10^{6} \\
\text { UC-MSCs, } 3 \text { cc of } \\
\text { saline with } 5 \% \text { A. } \\
\text { B. plasma. }\end{array}$ & $\begin{array}{l}\text { At } 12 \text {-month } \\
\text { assessment, Group-2 } \\
\text { patients reported } \\
\text { significant improvements } \\
\text { in knee function and } \\
\text { reduction in pain levels } \\
\text { when compared to the } \\
\text { Placebo group. No } \\
\text { differences in MRI } \\
\text { between treatment and } \\
\text { placebo group. No } \\
\text { severe AE reported. }\end{array}$ & $\begin{array}{l}\text { UC-MSC treatment } \\
\text { demonstrated safety } \\
\text { and clinical efficacy. }\end{array}$ & $\begin{array}{l}\text { Effects of the } \\
\text { treatment was } \\
\text { studied on a small } \\
\text { population. Repeated } \\
\text { trials involving a larger } \\
\text { population is } \\
\text { required. }\end{array}$ \\
\hline $\begin{array}{l}\text { Khalifeh- } \\
\text { Soltani S. } \\
\text { et } \text { al }^{31}\end{array}$ & $\begin{array}{l}20 \\
\text { (Age 35-75) }\end{array}$ & $\begin{array}{l}\text { Double-blind, } \\
\text { RCT }\end{array}$ & $\begin{array}{l}\text { Intra-articular } \\
\text { injections: } \\
\text { Placebo (saline only) } \\
\text { vs treatment group } \\
\left(0.5-0.6 \times 10^{8}\right) \\
\text { allogenic placenta } \\
\text { derived MSCs. }\end{array}$ & $\begin{array}{l}\text { Clinical improvements, } \\
\text { pain reduction, and } 10 \% \\
\text { increase in chondral } \\
\text { thickness observed in } \\
\text { treatment group after } \\
24-\text { weeks. Mild AEs } \\
\text { observed. }\end{array}$ & $\begin{array}{l}\text { Allogenic-placenta } \\
\text { derived MSC } \\
\text { demonstrated safety } \\
\text { and clinical efficacy } \\
\text { after } 24 \text { weeks } \\
\text { follow-up. }\end{array}$ & $\begin{array}{l}\text { Small sample size and } \\
\text { short-term study of } \\
\text { 24-weeks. }\end{array}$ \\
\hline $\begin{array}{l}\text { Park YB. } \\
\text { et al }{ }^{19}\end{array}$ & $\begin{array}{l}7 \\
\text { (Age 18-80) }\end{array}$ & $\begin{array}{l}\text { Open-label, } \\
\text { single-arm, } \\
\text { single center, } \\
\text { Phase I/II } \\
\text { uncontrolled } \\
\text { clinical trial }\end{array}$ & $\begin{array}{l}\text { Arthroscopy } \\
\text { procedure utilizing } \\
\text { Cartistem (H.A. gel+ } \\
\text { hUC-MSCs); } \\
\text { Group A (low dose- } \\
\text { I.I5-1.25 } \times 10^{7} \text { ) vs } \\
\text { Group B (high dose- } \\
\text { I.65-2.00x } 10^{7} \text { ) } \\
\text { followed over } 7 \text { years. }\end{array}$ & $\begin{array}{l}\text { Healed cartilage defect } \\
\text { and hyaline-like cartilage } \\
\text { seen after I year during } \\
\text { arthroscopic exam. } \\
\text { dGEMRIC showed } \\
\text { regenerated cartilage w/ } \\
\text { high GAG content at } 3 \\
\text { years. No significant } \\
\text { deterioration of } \\
\text { cartilage, and reduced } \\
\text { pain after } 7 \text { years. } \\
\text { Mild AEs observed in } \\
\text { patients during } \\
\text { treatment. }\end{array}$ & $\begin{array}{l}\text { First-in human clinical } \\
\text { trial utilizing } \\
\text { Cartistem } \\
\text { (composite of } \\
\text { culture-expanded } \\
\text { allogeneic hUCB- } \\
\text { MSCs and HA } \\
\text { hydrogel). The clinical } \\
\text { outcomes showed } \\
\text { improvement and } \\
\text { stability in the knee } \\
\text { joint for over seven } \\
\text { years. }\end{array}$ & $\begin{array}{l}\text { Further investigation } \\
\text { with a larger number } \\
\text { of patients is required } \\
\text { to study the efficacy } \\
\text { of Cartistem. This } \\
\text { study only evaluated } \\
\text { patients with grade-3 } \\
\text { KOA, and only two } \\
\text { patients consented to } \\
\text { arthroscopic } \\
\text { examination for } \\
\text { a biopsy a year post- } \\
\text { procedure. }\end{array}$ \\
\hline
\end{tabular}


- Section A: Patient demographics

- Section B: Pain and Function analysis - KOOS, VAS, \& WOMAC

The Knee Injury and Osteoarthritis Outcome Score (KOOS) is a knee-specific instrument that assesses the patient's opinion about their knee associated problems. There are five KOOS sub-scales which include Symptoms, Pain, Quality of Life (QoL), ADL \& Sport/ Recreation. KOOS is an extension of the WOMAC index, as it evaluates the short-term and long-term consequences of a knee injury. The KOOS scale is scored from 0 to 100; with 100 representing no knee problems, and zero representing extreme knee problems. ${ }^{16}$

Western Ontario and McMaster Universities Arthritis Index (WOMAC) is a questionnaire that evaluates the pain and function of the Hip and Knee osteoarthritis. ${ }^{16}$

Visual Analog Scale (VAS) is a unidimensional measure of pain intensity, and the scale runs $10 \mathrm{~cm}(100 \mathrm{~mm})$ in length both horizontally and vertically. A numeric score of zero indicates "no pain", and a score of one hundred indicates "severe/excruciating pain". 17

- Section C: Adverse Events and Radiographic analysis - Frequency, Severity of AEs \& MRI

Adverse Events (AEs) are defined as undesirable clinical outcomes that present post-intervention. AEs are graded based on severity: ${ }^{24}$

i. Mild: awareness of a symptom that does not affect the patient's activities of daily living (ADL) and is self-resolving. The use of simple analgesia helps reduce symptoms.

ii. Moderate: interferes with some patient's ADL and requires symptomatic care such as cold compression, regular analgesia, or opiate analgesia.

iii. Severe: significant discomfort affecting the patient's activity.

iv. Serious: can be a life-threatening or unexpected medical incident that requires hospitalization.

Magnetic Resonance Imaging (MRI) accurately assesses the cartilage thickness and volume to show morphological changes of the articular surface. Other changes to the internal cartilage signals and subchondral bone can easily be evaluated. Two main MRI imaging techniques will be focused on in this review; T2 and dGEMRIC. MRI T2 mapping enables a non-invasive measurement of the extracellular cartilage matrix by demonstrating changes in the collagen composition. $^{11,18}$ A delayed gadolinium-enhanced MRI of the cartilage (dGEMRIC) measures T1 relaxation time of the cartilage and tests the quality of the regenerated cartilage. ${ }^{19}$

\section{Bias Management}

To critically appraise the quality of literature assessment and data being analyzed for this review, a few precautionary measures were taken to minimize bias: ${ }^{20,21}$

- PRISMA-based flow chart and pre-specified criteria (search filters) were adopted to assess the eligibility criteria and ensure consistency of the studies being included $^{35}$

- reading the title and abstract and manually checking references of the studies that met the inclusion criteria but were excluded in this review; to reduce study-selection bias $^{35}$

- a reviewer working independently of the author revised each draft to reduce the risk of bias assessment

- rigorous double-checking on data extraction to reduce random errors and transcription errors (adding irrelevant data)

- heterogeneity between-study variations have been taken into account by summarizing all literature being reviewed into a single table in the Results section

The bias management technique adopted in this review answered a handful of essential signalling questions from a domain-based approach (ROBIS). ${ }^{20}$

\section{Results}

The online search through PubMed yielded 315 articles related to the treatment of KOA using Mesenchymal Stem Cells (MSCs), with the earliest article dating to July 2002. With the search filters applied, 291 publications were eliminated; this search excluded meta-analysis, books, documents, reviews, and abstracts. From the 24 human clinical trials, 3 studies were further excluded from our analysis (due to the use of cell-free treatments or stromal vascular fractions instead of MSCs). The clinical trials selected for this review were published between August 2015 and May 2019. The selection and elimination process of the studies in this review were demonstrated through a PRISMA-based flowchart in the Appendix (Figure S1).

Five hundred and eighty-nine patients participated in the 21 clinical trials, but not all patients in this review were formally diagnosed with KOA. Clinical trials using 
MSCs treatments on patients with Anterior Cruciate Ligament (ACL) injuries, Chondral lesions, damage to the menisci, and arthritic inflammations were also included in the review. KOA has a multifactorial etiology, and these isolated injuries are known causes to the development of radiographic KOA in injured patients. ${ }^{16}$ This review analyzes the efficacy of MSC therapy and investigates its effects on the treatment of KOA. The 21 clinical trials that use MSC therapies in the treatment of KOA are summarized in Table 1.

\section{Section A: Patient Demographics}

Patients enrolled in the clinical trials were aged between 18 and 80 years old, with the average patient age around 50 years old. Almost all of the patients enrolled in the clinical trials were overweight and had a BMI between 25 and 31. The only exception was for patients enrolled in the clinical trial conducted by Song et al; these patients had an average BMI that ranged between 23 and 25 .

In the clinical trial reviewed, 349 patients were formally diagnosed with KOA (Grade 2-4) and 257 patients had pre-existing knee joint defects that would eventually lead to radiographic KOA were included in the review. Tables A1-A4 in the appendix, breakdowns the number of patient's disease demographic and intervention assignment of all the clinical trials. This supplemental breakdown of information is essential, as it provides a clearer platform from which data can be distinguished and enables a more accurate analysis of the results achieved from the trials.

\section{Section B: Pain and Function Analysis (KOOS, VAS and WOMAC Scores) of MSC Treatments in Patients KOOS}

The improvement for KOOS scores per subscale at different dosages and frequencies of MSC treatments were compared in Figures 1-3. Table B1 in the appendix demonstrates the KOOS numerical values per subscale at baseline, 12-month follow up, and the improvement percentage.

Figures 1 and 2, both demonstrate that BM-derived and AD-derived MSCs result in subjective functional improvement and pain reduction at the knee joint of patients.
According to the figures, a treatment utilizing a lower concentration of MSC $\left(30.5 \times 10^{6}\right)$ on a more frequent basis (one dose at baseline and the second dose at 6-month/12month) is as effective as higher concentrations. The improvement percentages are comparable between a single-dose and double-dose at higher concentrations of MSC $\left(100 \times 10^{6}\right)$ interventions. Overall, both trials portray an improvement in the Activities of Daily Living (ADL), Quality of Life (QoL), sports and recreational activities, with a reduction in pain and symptoms.

Autologous MSCs in the clinical trials are derived either from the bone marrow or adipose tissue. Harvesting MSCs from the adipose tissue through liposuction provides a range of $20-60$ cells in $300 \mathrm{~mL}$ of adipose tissue. Five hundred times more cells when compared to bone marrow harvesting. ${ }^{32}$ Harvesting MSCs from bone marrow through posterior iliac aspiration is much more invasive than lipo-aspiration. ${ }^{32}$

Selecting a single preferable source of MSCs for future treatments proved to be a challenge because the trials conducted did not compare their efficacy individually or directly. The figures above cannot be used to compare the efficacy of MSCs from the adipose tissue versus the bonemarrow because the dosage is different. Further clinical investigation is required in order to focus on which derivation is more efficacious than the other. ${ }^{32}$

Intervention using an MSC combination IA injection (with Hyaluronan (HA) or Platelet-Rich Plasma (PRP)) is another form of MSC treatment that is being studied in various clinical trials. Bastos et al combine autologous BM-MSCs with PRP, and Wang et al combine Mesenchymal Progenitor Cells (MPCs) with Hyaluronan to better understand the impact of these additional factors on the efficacy of MSC treatment in the knee joint. The data in Table B2 (Appendix) extracted directly from Bastos et al illustrate the impact witnessed when MSC treatment was combined with PRP. ${ }^{28}$

Figure 3 demonstrates a positive outcome on pain reduction reported by patients in the MSC+PRP combination treatment group when compared to the MSC only treatment group. Therefore, indicating that the combination of treatment of MSCs with PRP is safe. Preliminary data indicate no significant clinical outcome differences between both treatment groups. Limitations, seen in Bastos et al, were the lack of a PRP-only control group and a small sample population of 18 patients only. It is important to monitor the effects of PRP only (control group) from the baseline to a 12-month follow-up period, 


\section{Improvement of KOOS (\%), Al Najar, et al}

140.0

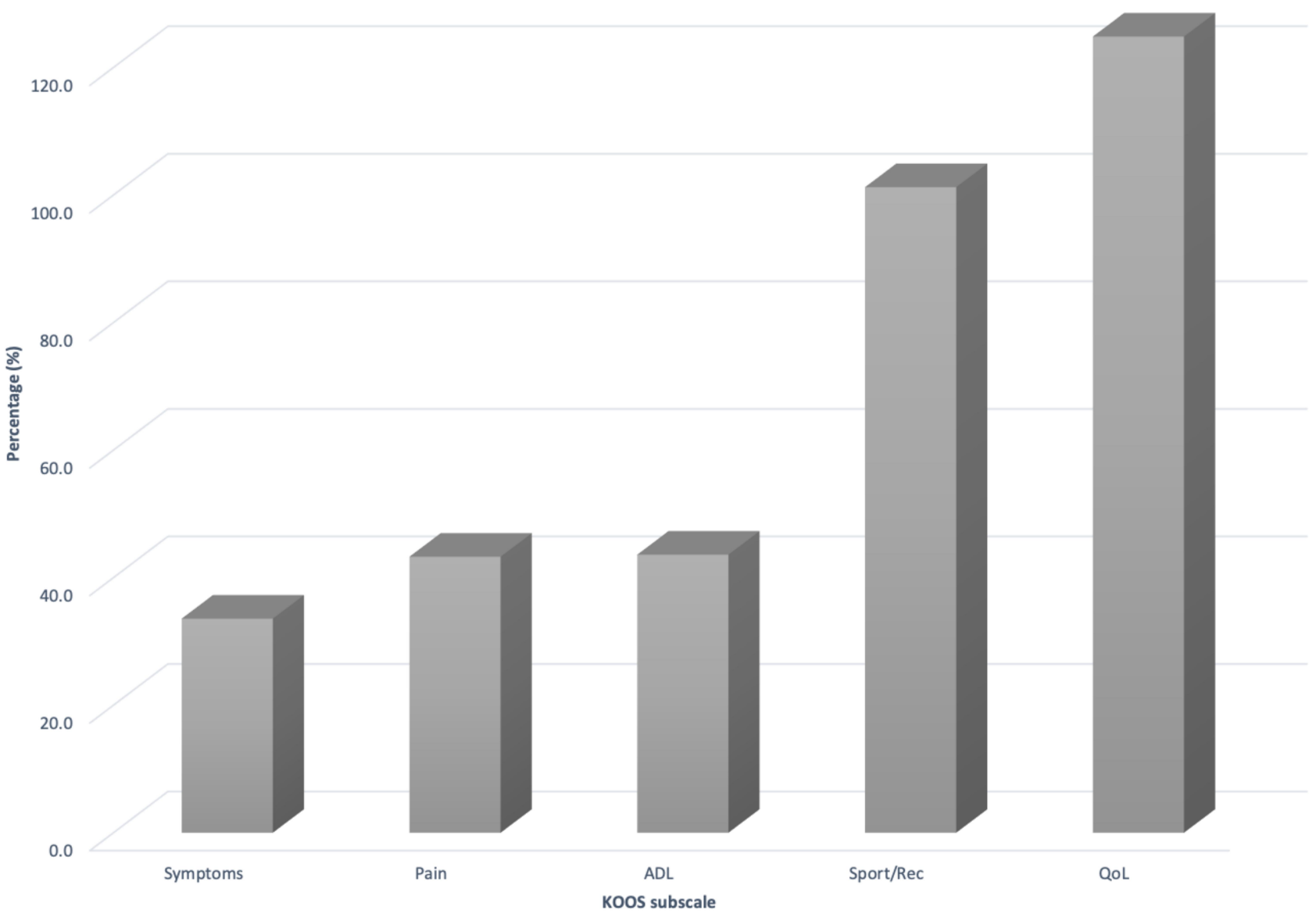

Figure I Percentage of KOOS scale improvement per subscale post-MSC intervention $\left(30.5 \times 10^{6}\right.$ Autologous BM-MSCs and double-dose).

to allow for better comparison and data analysis with other treatment groups.

The clinical trial conducted by Wang et al compares HA control group versus MPC + HA treatment group over a 24-month follow-up period. ${ }^{7}$ Both groups demonstrated a positive improvement from baseline. Significant improvement in all KOOS subscales was observed in the MPC+HA treatment group when compared to the HA only group. There was a significant improvement in function through ADL $(p=0.04)$, and reduction in pain/symptoms $(\mathrm{p}=0.03)$ at the 18 -month follow-up period.

Other trials have also combined MSC therapies along with surgical/arthroscopic interventions in patients with a severe cartilage defect of the knee. Clinical trials that have combined arthroscopic intervention with MSC implantation in this review include; Shetty et al, Park et al, Koh et al, Hernigou et al, and Freitag et al.

Shetty et al combined arthroscopic microfracture procedure with Mesenchymal Cell-Induced Chondrogenesis (MCIC) that was obtained from the bone marrow of patients and achieved an overall KOOS improvement from 64.5 to $>89.2(\mathrm{P}<0.05)$. Autologous chondrocyte implantation (ACI) and matrix-induced autologous chondrocyte (MACI) are cell therapies that are available for the treatment of focal cartilage defects. Unfortunately, these therapies are not as effective at treating KOA because the cartilage defects are large and show non-contained pathogenesis. Further studies are being conducted to better understand the impact of MCIC on larger cartilage defects. ${ }^{12,25}$ 
100

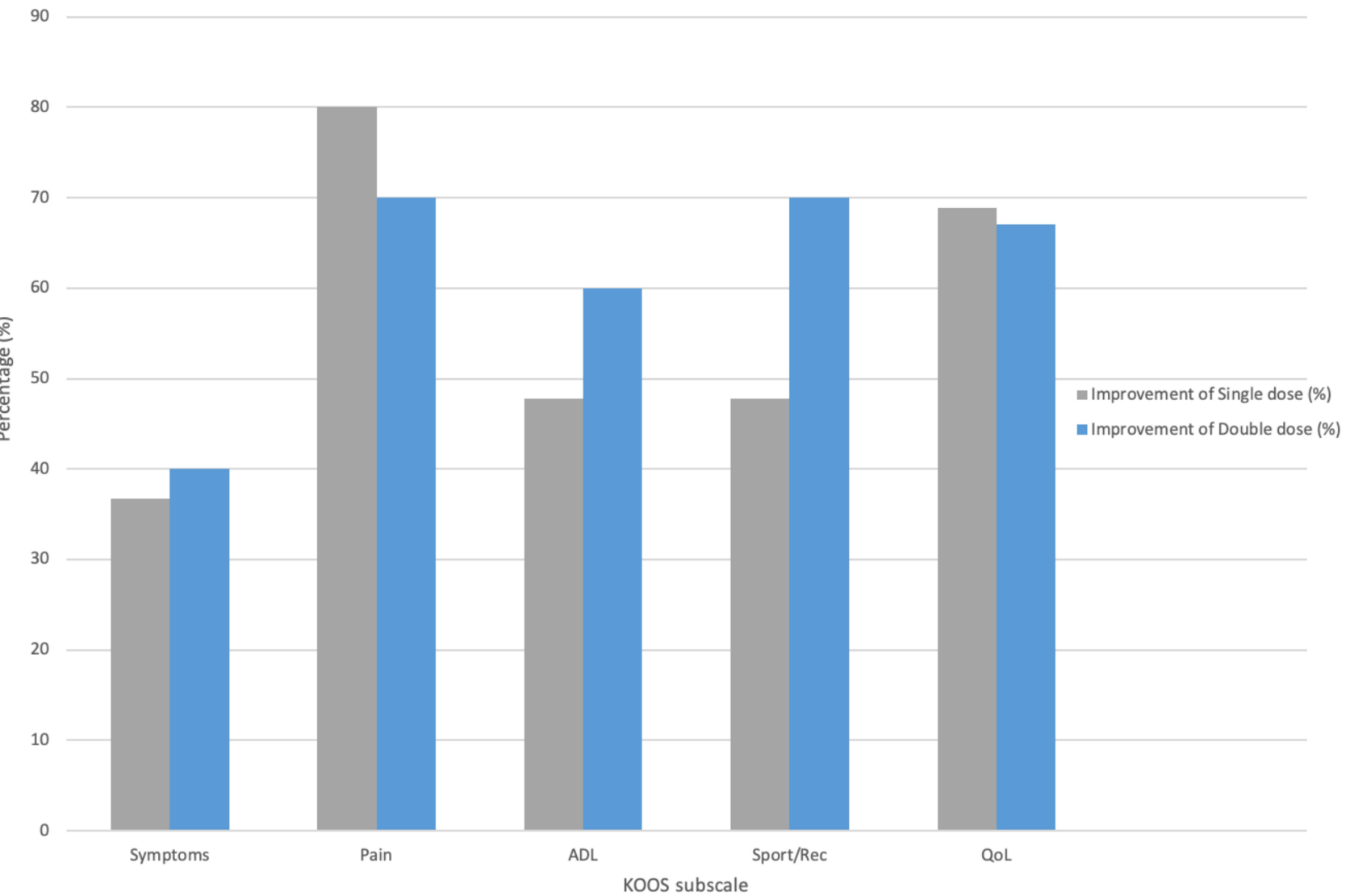

Figure 2 Percentage of KOOS scale improvement per subscale post-MSC intervention ( $100 \times 10^{6}$ Autologous AD-MSCs, single-dose versus double-dose).

Although the improvement observed in most trials did not demonstrate statistical significance, the overall impact of treatments utilizing MSCs indicates an improvement in all KOOS subscales, especially in pain reduction and function.

\section{VAS}

VAS is another tool that can be used to study the impact of MSC therapy on pain at the knee joint. Four studies involving the use of MSCs via IA injections using various concentrations and origins of MSCs (allogenic vs autologous) were compared in Table B3 (Appendix section) and Figure 4.

The first six concentrations of the intervention dosage belong to Gupta et al, allogenic BM-MSCs /aBM-MSCs $\left(25 \times 10^{6}\right.$ MSCs, $50 \times 10^{6}$ MSCs, $75 \times 10^{6}$ MSCs, $150 \times 10^{6}$ MSCs, Placebo $1-2 \mathrm{~mL}$ and Placebo $2-2 \mathrm{~mL}$ ), then Pers et al, autologous adipose-derived MSCs $\left(2 \times 10^{6} \mathrm{MSCs}, 10 \times 10^{6} \mathrm{MSCs}, 50 \times 10^{6} \mathrm{MSCs}\right)$, then Lamo-Espinosa et al, autologous bone-marrow MSCs (control HA - $4 \mathrm{~mL}, 10 \times 10^{6}$ MSC, $100 \times 10^{6}$ MSC) and finally, Park et al, CARTISTEM- umbilical cord-MSCs $\left(50 \times 10^{6} \mathrm{MSCs}\right)$.

According to Table B3 (Appendix) and Figure 4, intervention dosage between $2 \times 10^{6}$ MSCs - $50 \times 10^{6}$ MSCs, provides patients with the most pain relief and has an optimal efficacy when compared to doses above $50 \times 10^{6}$ MSCs. Dosages above $50 \times 10^{6}$ MSCs result in a decline of VAS, which correlates to less pain reduction perceived by patients at the knee joint.

Figure 4 shows a trend that lower concentrations of autologous MSCs can be used to reach similar levels of VAS improvement as higher concentrations of allogeneic MSCs. Autologous BM-MSCs $\left(10 \times 10^{6}\right)$ at $71.4 \%$ efficacy when compared to allogenic BM-MSCs $\left(25 \times 10^{6}\right)$ at $66.2 \%$. More clinical trials with a larger cohort are required to compare the most efficacious origin of MSCs 


\section{Improvement of KOOS (\%), Bastos, et al}

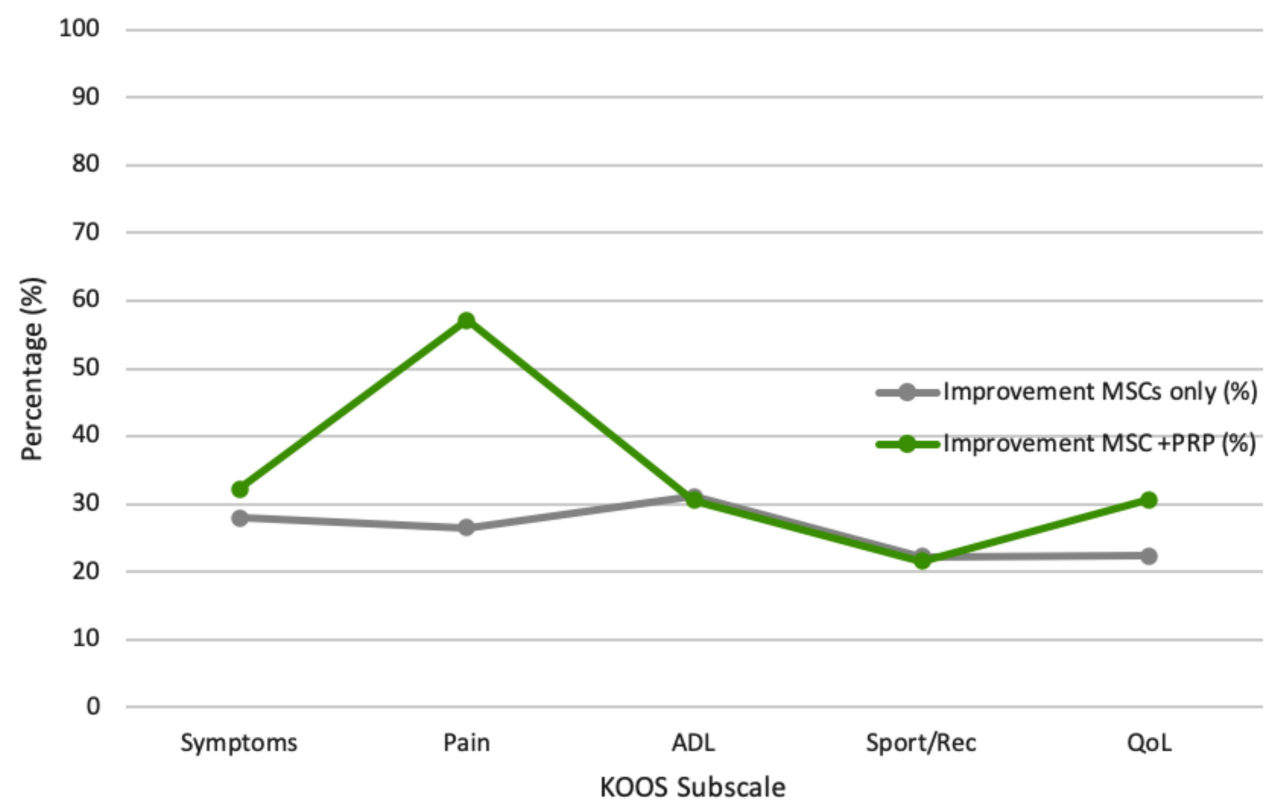

Figure 3 Percentage of KOOS scale improvement per subscale post-MSC intervention (BM-MSCs vs BM-MSCs + PRP).

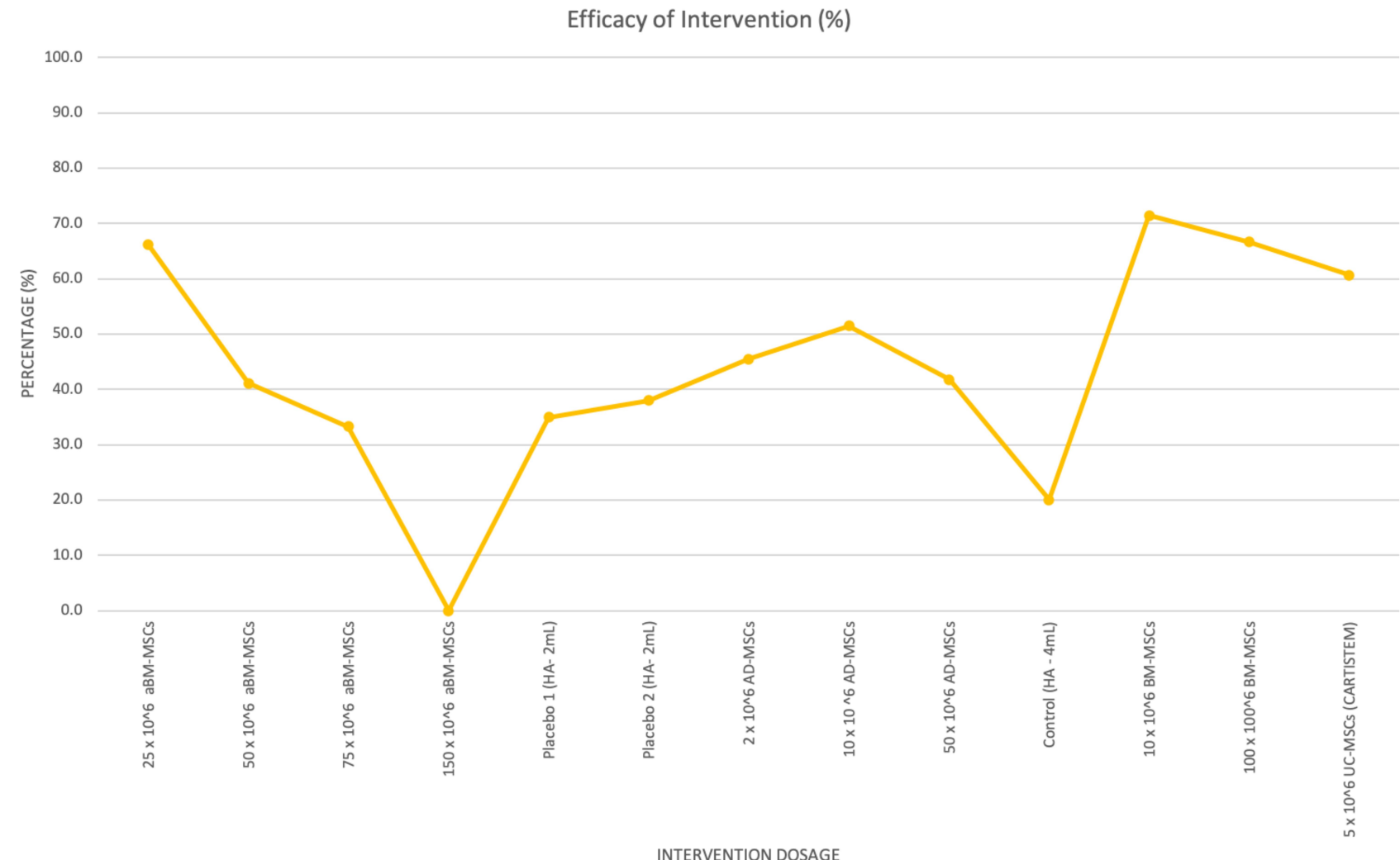

Figure 4 Percentage of intervention efficacy against MSC dosage and origin on VAS.

and support this assumption with certainty. Patients in the Compared to the placebo group, there is no statistical control/placebo group receiving Hyaluronic acid significance in pain reduction in VAS observed in the (2-4 mL) are also experiencing some pain relief. treatment group. 


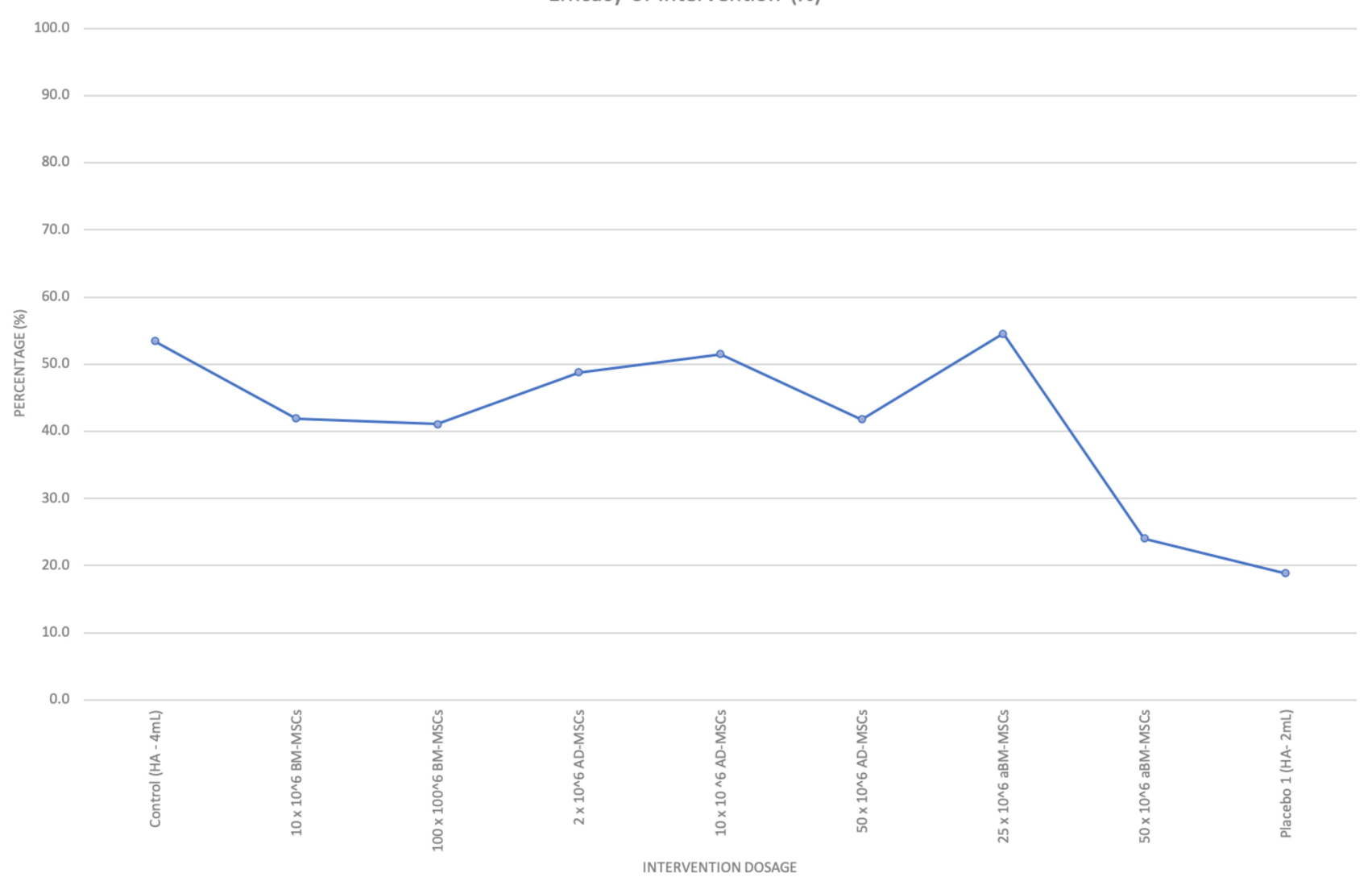

Figure 5 Percentage of intervention efficacy against MSC dosage and origin on WOMAC index.

\section{WOMAC}

The WOMAC index is also utilized to monitor the pain, stiffness, and function of the knee joint.

Table B4 (Appendix) and Figure 5, compare WOMAC results and the intervention efficacy achieved from three different studies.

The first 3 concentrations of the intervention dosage belong to Lamo-Espinosa et al, autologous BMMSCs (control HA $-4 \mathrm{~mL}, 10 \times 10^{6}$ MSCs, $100 \times 10^{6}$ MSCs), then Pers et al, autologous adipose-derived MSCs $\left(2 \times 10^{6}\right.$ MSCs, $10 \times 10^{6}$ MSCs, $50 \times 10^{6}$ MSCs), and finally Gupta et al, allogenic BoneMarrow MSCs $\left(25 \times 10^{6}\right.$ MSCs, $50 \times 10^{6}$ MSCs, and Placebo1 - 2mL).

High dosage treatment groups from Gupta et al showed a sharp decline in treatment efficacy and were not included in Table B4 and Figure 5. Doses between $2 \times 10^{6}$ MSCs $50 \times 10^{6}$ MSCs are efficacious but not statistically significant when compared to the results achieved in the control group. Figure 3 does not show a clear trend on the impact of the origin of MSCs (autologous vs allogeneic) on the efficacy of intervention.

\section{Section C: Adverse Events and Radiographic Analysis (MRI) of MSC Treatments in KOA Patients \\ Adverse Events}

Monitoring the frequency and types of AEs observed during the follow-up periods of the intervention is essential to understanding the safety and tolerability of MSC treatments. Table 2 identifies the AEs mentioned in the clinical trials being studied in this review.

IA Injections are the most common form of MSC delivery method; with 250 patients placed in the treatment group and 94 patients placed in the control group (HA or Saline only). Table 2 shows higher accounts of AEs at the site of injection administration and musculoskeletal tissue for the treatment group when compared to the control group. Arthroscopic interventions combined with MSC treatments 
Table 2 Identifies the Manifestation of the Adverse Events Observed During the Follow-Up Periods

\begin{tabular}{|c|c|c|}
\hline Type of Adverse Events & Frequency of A.E.'s in MSC Treatment & Frequency of A.E.'s in Control \\
\hline Blood \& Lymphatic system disorder & I & I \\
\hline Endocrine disorders & 2 & 0 \\
\hline Eye disorder & I & 0 \\
\hline Gastrointestinal disorders & 4 & 5 \\
\hline General disorder and site of Injection Administration & 84 & 27 \\
\hline Infection \& Infestation & 14 & 7 \\
\hline Injury, Procedural \& Poisoning & 11 & 3 \\
\hline Investigation & 4 & 3 \\
\hline Metabolism \& Nutrition disorders & 4 & 4 \\
\hline Musculoskeletal \& Connective tissue & 72 & 20 \\
\hline Nervous system disorders & 2 & I \\
\hline Urinary and renal disorders & 2 & 0 \\
\hline Reproductive system disorders & 2 & 0 \\
\hline Respiratory, Thoracic \& Mediastinal disorders & 1 & I \\
\hline Skin \& Subcutaneous tissue disorder & I & $\mathrm{I}$ \\
\hline Surgical \& Medical procedure complications & 3 & 0 \\
\hline Vascular disorders & 3 & 2 \\
\hline Immune System & 2 & 1 \\
\hline Total \# of AEs & 213 & 76 \\
\hline
\end{tabular}

correspond to higher accounts of procedural injury and infection observed in the treatment group.

It is important to note that there were more patients enrolled in the treatment group than in the control group, so the significantly higher accounts of AEs observed can be attributed to the imbalance in the number of patients enrolled in each group. Not all the AEs can be attributed to the intervention received; age, high BMI, and other preexisting chronic conditions in patients account for some AEs recorded over the follow-up periods.

The number of AEs and the severity of the AEs (Mild, Moderate, and Severe) correlating to the intervention dosage, frequency, and origin of MSC received in five different studies are compared to Figure 6. The data of each intervention per the severity of AE are also presented in Table C1 (Appendix section).

Figure 6 shows an increase of AEs incidence and severity when the dosage of the IA injection exceeds (50 $\left.x 10^{6}\right)$. When the frequency of the IA injections increases, the number of AEs increases, and the severity of the AEs shift from mild to moderate. No trend can be seen in Figure 6 when comparing various MSC origins (autologous vs allogeneic) to the number of AEs observed. The control group using Hyaluronic acid showed seven instances of mild AEs.

Table C1 (Appendix) and Figure 6; show the rarity of observing serious life-threatening AEs post-intervention, deeming MSCs from various origins (autologous vs allogeneic), doses, and frequencies used in the clinical trials as tolerable and safe. According to the literature, most AEs observed were local swelling at the site of injection and moderate arthralgia that subsided within 24-48 hrs following the intervention. ${ }^{7,11}$ Cold compression and mild oral analgesia were used to relieve the pain. No clinical and biochemical AEs were observed in long-term follow-up periods. ${ }^{24}$

\section{Radiological Assessment - MRI}

Radiological assessment is necessary to demonstrate whether the goal of restoring and regenerating cartilage at the knee joint is met when using MSCs. Most of the clinical trials used various MRI scanning techniques such as dGEMRIC and T2 mapping, and MRI scoring tools such as MOCART \& MOAKS to assess the restoration of cartilage at the knee joint. The variety of results achieved makes it challenging for comparison. Davatchi et al and Vega et al refer to the improvement of the cartilage thickness as stabilization of cartilage degeneration at the site of OA followed by early signs of MSCs differentiation into chondrocytes and cartilage regrowth.

Table C2 (Appendix) and Figure 7 both aim to compare and show the improvement seen in cartilage thickness in three different clinical trials. 


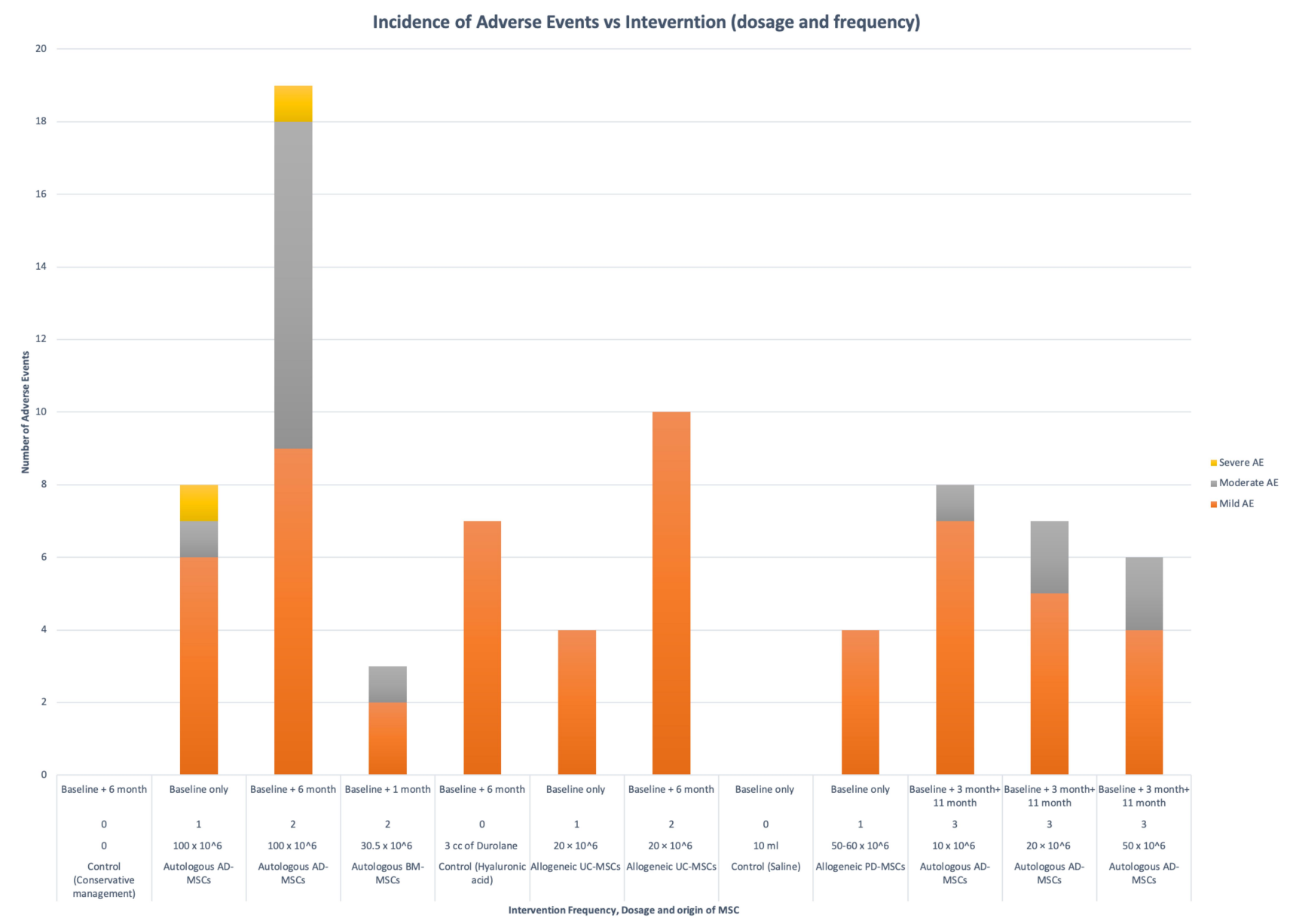

Figure 6 Incidence of AEs versus intervention dosage and frequency.

The MRI radiological assessments measure the width of mean joint space (overall femoral and tibial condyles, from the lateral to the medial points) in a sagittal plane at baseline and the specified follow-up period of 6,12 , or 24 months depending on the clinical trial. ${ }^{7,14}$

Both frequent lower concentration $\left(30.5-60 \times 10^{6}\right)$ and high concentration $\left(100 \times 10^{6}\right)$ of IA injections containing MSCs seem to be efficacious in stabilizing cartilage degeneration and improving the cartilage thickness. More research focused on comparing the frequency, and the concentration of MSCs in IA injections is required to provide a better understanding of the optimal treatment.

Other clinical trials that focused on cartilage thickness of the knee-joint observed positive but not statistically significant results when compared to the control group. Twenty-six (65\%) patients receiving arthroscopic intervention combined with MSC treatment in Koh et al showed hypertrophic cartilage coverage of the lesions and 32 $(80 \%)$ patients exhibited normal repair tissue signals compared to the control group $18(40 \%)$ patients. In the clinical trial conducted by PERS et al, only half the patients demonstrated limited positive changes in the cartilage thickness. Due to the small number of patients enrolled (six patients), a correlation between the MRI and improvement in clinical outcome was not observed. ${ }^{26}$

According to the trial conducted by Vega et al, significant improvements were reported between the 6th and 12th month of the MSC treatment group when compared to a lesser improvement observed in the active control group. Overall, these clinical trials show encouraging results in terms of pain relief, improved function at the knee joint, and an increase of signals pointing to tissue repair.

The clinical trials reviewed are extremely heterogenous. Beginning with patient demographic, experimental design, and the collection of calculations used to report data. There is not a standardized form or consensus between researchers on reporting data in clinical trials. KOOS, VAS \& WOMAC are quantitative results achieved from subjectively rating scales/ 
Improvement in Cartilage thickness (\%)

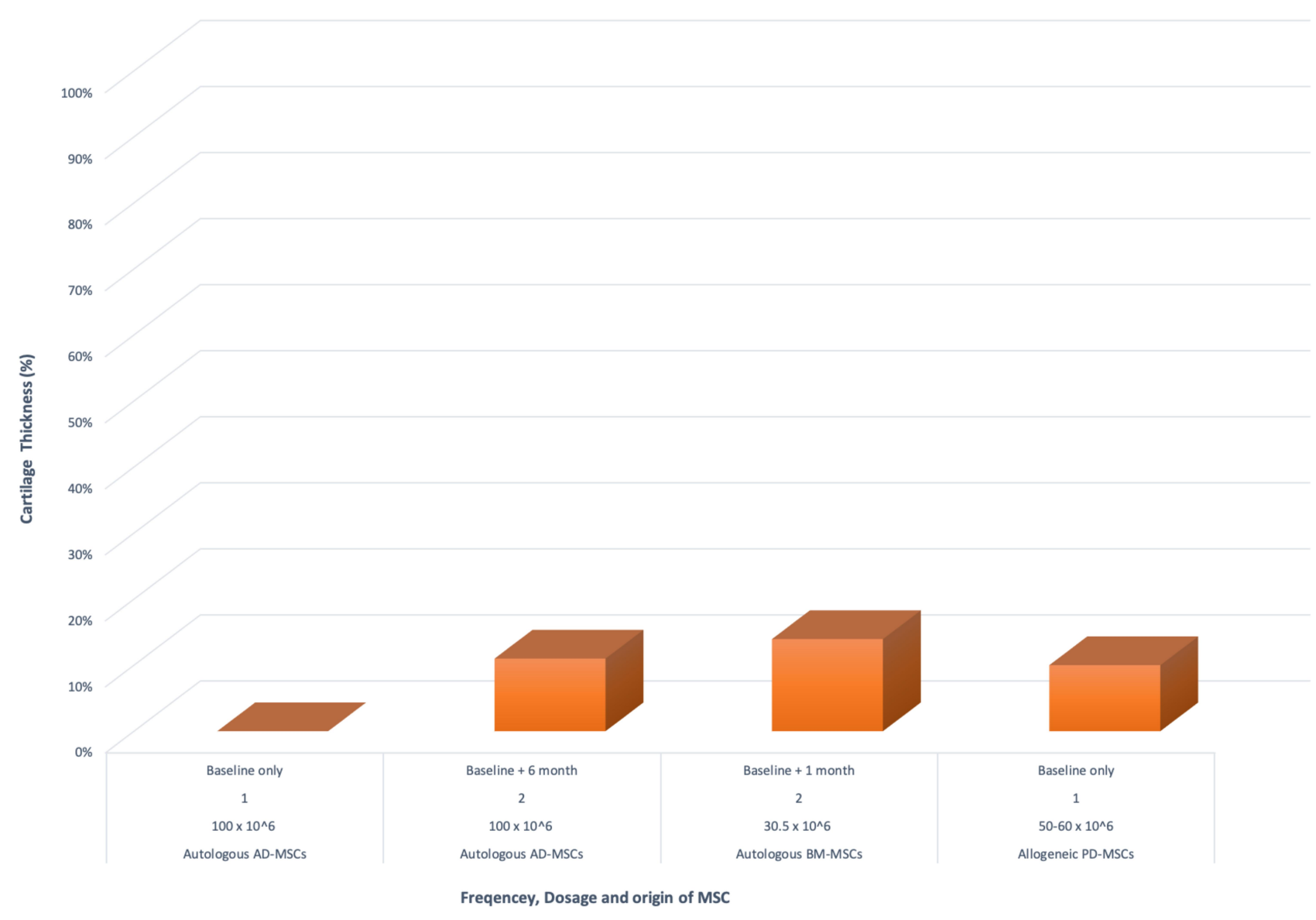

Figure 7 Percentage of improvement of cartilage thickness post-MSC intervention when compared to dosage and frequency of treatment.

questionnaires. It is important to understand that the large efficacy percentages, seen in Figures 1-5, are only used to compare subjective trends experienced by patients in a few trials and not the actual efficacy of the intervention itself.

The true "efficacy of intervention" is the result achieved from the objective quantitative assessment during the follow-up period. This assessment includes Frequency \& Severity of AEs, MRI, histological, and X-ray results. Although both subjective and objective quantitative results show a trend towards clinical efficacy, the results from the trials are not statistically significant.

\section{Discussion}

All clinical trials included in this review demonstrated the safety and feasibility of MSC treatments. Since there were no changes to pathological values obtained from T2 mapping in healthy regions of the joint during long-term followup periods, this is suggestive of disease stabilization. ${ }^{1,11}$ Subjective parameters such as KOOS, VAS \& WOMAC demonstrate pain reduction and improved function at the knee joint from baseline through post-intervention followup. The improved subjective results were not statistically significant in most trials. The frequency/severity of adverse events and the radiological assessment through an MRI are the objective parameters assessed in this review. A regenerative pattern of neo-cartilage formation was also seen from 24 weeks to 24 months post-intervention. The efficacy of the intervention can be further improved if MSC implantation occurs at an earlier stage rather than an advanced stage of KOA. ${ }^{9}$ There was no evidence of ectopic tissue growth or tumor formation locally at a 1-year followup. Haematological, biochemical, and serological parameters were comparable in both the stem cell and placebo arm in all groups of patients.

\section{Dosage and Frequency}

The optimal therapeutic dosage of MSCs in the treatment of KOA is still not clear. The current tested doses from the 
clinical trials reviewed range from $2 \times 10^{6}$ to $150 \times 10^{6}$ MSCs. According to Gupta et al, high-dose groups $(\geq 50$ $\left.\mathrm{x} 10^{6} \mathrm{MSCs}\right)$ are correlated with efficacy but increased instances of AEs, when compared to low dose and control groups. The more optimal and efficacious form of treatment that demonstrated improvement in all subjective parameters was seen in the $25 \times 10^{6}$ MSCs treatment group. Yet, the results were still not statistically significant. Trials conducted by Song et al, Al-Najar et al, and Mastas et al showed that concentrations between $2 \times 10^{6}$ and $60 \times 10^{6} \mathrm{MSCs}$, when applied more frequently to the site of the disease were potentially within the range of optimal efficacy for MSC intervention.

The clinical trial conducted by Bastos et al demonstrated safety but no additional clinical benefit to combining MSC with or Platelet Rich Plasma (PRP). The action of PRP alone on the regeneration of articular cartilage has been widely studied because of its inherent potential of being a biological treatment for KOA. PRP is derived directly from the patient's blood where the platelets are concentrated and activated ex-vivo, releasing high concentrations of growth factors (such as TGF- $\beta$, FGF, and IGF) all of which have important chondrogenic properties. Bastos et al became one of the first clinical trials to study the effectiveness of MSC treatment when combined with PRP. This trial showed improved efficacy in pain and function when compared to MSC only treatment. ${ }^{28}$

MSC treatments combined with Hyaluronic acid (HA) were observed to slightly improve the efficacy of MSCs treatments by increasing the rate of engraftment and chondrogenic activity. ${ }^{15}$ Clinical trials that combine MSC treatments with HA include; Gupta et al, Lamo-Espinosa et al, Vega et al, and Wang et al. The HA found in the joints of patients with advanced-stage KOA tend to be depolymerized into a lower-molecular-weight compound that has a weakened mechanical and viscoelastic properties in the synovial fluid. Exogenous HA can be used as a form of treatment. It can be taken orally or via IA injections. HA helps with maintaining the synovial fluidity by acting as a lubricant, scavenging free radicals, inducing chondroprotective and anti-inflammatory effects. MSC-HA combination treatments demonstrated the potential in maximizing the effects of MSC interventions. ${ }^{33}$

\section{Origin of MSC}

MSCs can be derived from allogenic sources (human donor-derived MSCs umbilical cord, placenta, adipose, or bone-marrow) or autologous sources (patients derived MSCs from either the bone marrow or the adipose tissue). ${ }^{34}$

Allogenic sources of MSCs are now being studied more frequently. Their wide availability, convenience, lower cost, higher homogeneity, and the possibility of using it in seropositive patients ${ }^{14}$ allow for its broader application. Allogenic sources of MSCs do not require invasive procedures to extract MSCs when compared to autologous procedures. Deriving autologous MSCs is not cost-effective and is a two-step procedure that involves a harvesting step and a transplanting step. For example, the harvesting step from the bone marrow requires the patient to undergo bone marrow aspiration from the posterior iliac crest. This has to take place in an operating room under local anaesthesia, which is slightly invasive and time-consuming. ${ }^{11}$ The harvesting step from the adipose tissue requires the patients to undergo an outpatient liposuction procedure under local anaesthesia. The autologous stem cells are then prepared and expanded at a single Good Manufacturing Practice (GMP) facility, typically delivered within a period of 2 weeks. ${ }^{34}$

When studied for its therapeutic safety and efficacy, the impact of allogenic MSCs was somewhat comparable yet less efficacious than autologous sources. Emadedine et al mentioned that allogenic MSCs tend to produce a greater increase in the peak of inflammation $24 \mathrm{hr}$ post-intervention when compared to patients receiving MSCs from autologous sources. According to Vega et al, the healing effects (such as anti-inflammatory and cartilage regeneration) of allogenic MSCs are less than autologous MSCs.

Vega et al caution researchers to ensure safety when transitioning from autologous to allogeneic sources. Further research is required to address how allogenic MSCs overcome the immune system and show efficacy when promoting cartilage repair. Two stem cell-based medicinal products have been created from allogenic sources and studied in this review: Cartistem (UC-MSCs) by Park et al and Stempeucel (BM-MSCs) by Gupta et al. Long-term clinical outcomes demonstrate stabilization of cartilage degradation with no significant AEs (such as tumorigenesis) observed. ${ }^{19}$ The procedure of deriving allogenic MSCs through cell handling, expanding, and quality control is reproducible and satisfactory.

\section{Conclusion}

Interventions using MSCs have shown a trend towards clinical efficacy and validity as a form of treatment, yet 
the clinical outcomes were not statistically significant nor optimal. MSC treatments are a superior approach to conventional treatments. They take on a path of OA stabilization by reducing inflammation, followed by the restoration of damaged cartilage tissue. Encountering serious local or systemic adverse events from MSC treatments seems to be highly improbable, suggesting its "hypo immunogenic" benefits and tolerability. Most trials have shown a peak in positive effects between the 6th and12th month of treatment. 9,14

Nonetheless, many questions regarding the mechanism of MSCs remain unanswered, given the limited number of RCTs conducted. This makes it a challenge to form solid conclusions on the overall efficacy and safety of MSCs. There is a justified need for further investigations (preferably RCTs) over extended periods with a larger cohort to assess the impact of MSCs. Future MSC treatment trials should take into account; treatments in early-stage KOA patients (K/L Grade 0-2), the cell source (efficacy of allogenic vs autologous), the dosage, the frequency of therapy, MSC combined treatments (w/co-activators such as HA or PRP), and the therapy delivery method (arthroscopic/surgical implantation vs IA injection).

\section{Disclosure}

The authors declare no conflicts of interest.

\section{References}

1. Song Y, Du H, Dai C, et al. Human adipose-derived mesenchymal stem cells for osteoarthritis: a pilot study with long-term follow-up and repeated injections. Regen Med. 2018;13(3):295-307. doi:10.22 17/rme-2017-0152

2. Zhang R, Ma J, Han J, Zhang W, Ma J. Mesenchymal stem cell-related therapies for cartilage lesions and osteoarthritis. Am J Transl Res. 2019;11(10):6275-6289.

3. Anandacoomarasamy A, March L. Current evidence for osteoarthritis treatments. Ther Adv Musculoskelet Dis. 2010;2:17-28. doi:10.1177/ $1759720 X 09359889$

4. Losina E, Paltiel AD, Weinstein AM, et al. Lifetime medical costs of knee osteoarthritis management in the United States: impact of extending indications for total knee arthroplasty. Arthritis Care Res. 2015;67 (2):203-215. doi:10.1002/acr.22412

5. Freitag J, Bates D, Wickham J, et al. Adipose-derived mesenchymal stem cell therapy in the treatment of knee osteoarthritis: a randomized controlled trial. Regen Med. 2019;14(3):213-230. doi:10.2217/rme2018-0161

6. Zhang Y, Jordan JM. Epidemiology of osteoarthritis [published correction appears in Clin Geriatr Med. 2013 May;29(2):ix]. Clin Geriatr Med. 2010;26(3):355-369. doi:10.1016/j.cger.2010.03.001

7. Wang Y, Shimmin A, Ghosh P, et al. Safety, tolerability, clinical, and joint structural outcomes of a single intra-articular injection of allogeneic mesenchymal precursor cells in patients following anterior cruciate ligament reconstruction: a controlled, double-blind, randomized trial. Arthritis Res Ther. 2017;19(1):180. doi:10.1186/s13075017-1391-0
8. Ashkavand Z, Malekinejad H, Vishwanath BS. The pathophysiology of osteoarthritis. J Pharm Res. 2013;7:132-138. doi:10.1016/j.jopr. 2013.01.008

9. Davatchi F, Sadeghi Abdollahi B, Mohyeddin M, Nikbin B. Mesenchymal stem cell therapy for knee osteoarthritis: 5 years follow-up of three patients. Int J Rheum Dis. 2016;19(3):219-225. doi:10.1111/1756-185X.1267

10. Freitag J, Ford J, Bates D, et al. Adipose-derived mesenchymal stem cell therapy in the treatment of isolated knee chondral lesions: design of a randomized controlled pilot study comparing arthroscopic microfracture versus arthroscopic microfracture combined with postoperative mesenchymal stem cell injections. BMJ Open. 2015;5(12): e009332. doi:10.1136/bmjopen-2015-009332

11. Soler R, Orozco L, Munar A, et al. Final results of a Phase I-II trial using ex vivo expanded autologous mesenchymal stromal cells for the treatment of osteoarthritis of the knee confirming safety and suggesting cartilage regeneration. Knee. 2016;23(4):647-654. doi:10. 1016/j.knee.2015.08.013

12. Emadedin $\mathrm{M}$, Labibzadeh $\mathrm{N}$, Liastani $\mathrm{MG}$, et al. Intra-articular implantation of autologous bone marrow-derived mesenchymal stromal cells to treat knee osteoarthritis: a randomized, triple-blind, placebo-controlled Phase 1/2 clinical trial. Cytotherapy. 2018;20 (10):1238-1246. doi:10.1016/j.jcyt.2018.08.005

13. Shadmanfar S, Labibzadeh N, Emadedin M, et al. Intra-articular knee implantation of autologous bone marrow-derived mesenchymal stromal cells in rheumatoid arthritis patients with knee involvement: results of a randomized, triple-blind, placebo-controlled phase $1 / 2$ clinical trial. Cytotherapy. 2018;20(4):499-506. doi:10.1016/j.jcyt. 2017.12.009

14. Vega A, Martín-Ferrero MA, Del Canto F, et al. Treatment of knee osteoarthritis with allogeneic bone marrow mesenchymal stem cells: a randomized controlled trial. Transplantation. 2015;99(8):16 81-1690. doi:10.1097/TP.0000000000000678

15. Gupta PK, Chullikana A, Rengasamy M, et al. Efficacy and safety of adult human bone marrow-derived, cultured, pooled, allogeneic mesenchymal stromal cells $\left(\right.$ Stempeuce ${ }^{\circledR}$ ): a preclinical and clinical trial in osteoarthritis of the knee joint. Arthritis Res Ther. 2016;18 (1):301. doi:10.1186/s13075-016-1195-7

16. Roos EM, Lohmander LS. The Knee injury and Osteoarthritis Outcome Score (KOOS): from joint injury to osteoarthritis. Health Qual Life Outcomes. 2003;1:64. doi:10.1186/1477-7525-1-64

17. Bolognese JA, Schnitzer TJ, Ehrich EW. Response relationship of VAS and Likert scales in osteoarthritis efficacy measurement. Osteoarthritis Cartilage. 2003;11(7):499-507. doi:10.1016/s10634584(03)00082-7

18. Gold GE, Chen CA, Koo S, Hargreaves BA, Bangerter NK. Recent advances in MRI of articular cartilage. AJR Am J Roentgenol. 2009;193(3):628-638. doi:10.2214/AJR.09.3042

19. Park YB, Ha CW, Lee CH, Yoon YC, Park YG. Cartilage regeneration in osteoarthritic patients by a composite of allogeneic umbilical cord blood-derived mesenchymal stem cells and hyaluronate hydrogel: results from a clinical trial for safety and proof-of-concept with 7 years of extended follow-up. Stem Cells Transl Med. 2017;6 (2):613-621. doi:10.5966/sctm.2016-0157

20. Whiting P, Savović J, Higgins JP, et al. ROBIS: A new tool to assess risk of bias in systematic reviews was developed. J Clin Epidemiol. 2016;69:225-234. doi:10.1016/j.jclinepi.2015.06.005

21. Almeida CP, Goulart BN. How to avoid bias in systematic reviews of observational studies. Revista CEFAC. 2017;19(4):551-555. doi:10.1590/1982-021620171941117

22. Lamo-Espinosa JM, Mora G, Blanco JF, et al. Intra-articular injection of two different doses of autologous bone marrow mesenchymal stem cells versus hyaluronic acid in the treatment of knee osteoarthritis: multicenter randomized controlled clinical trial (phase I/II). J Transl Med. 2016;14(1):246. doi:10.1186/s12967-016-0998-2 
23. Lamo-Espinosa JM, Mora G, Blanco JF, et al. Intra-articular injection of two different doses of autologous bone marrow mesenchymal stem cells versus hyaluronic acid in the treatment of knee osteoarthritis: long-term follow up of a multicenter randomized controlled clinical trial (phase I/II). J Transl Med. 2018;16(1):213. doi:10.1186/s12967018-1591-7

24. Al-Najar M, Khalil H, Al-Ajlouni J, et al. Intra-articular injection of expanded autologous bone marrow mesenchymal cells in moderate and severe knee osteoarthritis is safe: a phase I/II study. J Orthop Surg Res. 2017;12(1):190. doi:10.1186/s13018-017-0689-6

25. Shetty AA, Kim SJ, Ahmed S, Trattnig S, Kim SA, Jang HJ. A cost-effective cell- and matrix- based minimally invasive single-stage chondroregenerative technique developed with validated vertical translation methodology. Ann R Coll Surg Engl. 2018;100 (3):240-246. doi:10.1308/rcsann.2017.0223

26. Pers YM, Rackwitz L, Ferreira R, et al. Adipose mesenchymal stromal cell-based therapy for severe osteoarthritis of the knee: a phase i dose-escalation trial. Stem Cells Transl Med. 2016;5 (7):847-856. doi:10.5966/sctm.2015-0245

27. Hernigou P, Auregan JC, Dubory A, Flouzat-Lachaniette $\mathrm{CH}$, Chevallier N, Rouard H. Subchondral stem cell therapy versus contralateral total knee arthroplasty for osteoarthritis following secondary osteonecrosis of the knee. Int Orthop. 2018;42(11):2563-2571. doi:10.1007/s00264-018-3916-9

28. Bastos R, Mathias M, Andrade R, et al. Intra-articular injections of expanded mesenchymal stem cells with and without addition of platelet-rich plasma are safe and effective for knee osteoarthritis. Knee Surg Sports Traumatol Arthrosc. 2018;26(11):3342-3350. doi:10.1007/s00167-018-4883-9

29. Koh YG, Kwon OR, Kim YS, Choi YJ, Tak DH. Adipose-derived mesenchymal stem cells with microfracture versus microfracture alone: 2-year follow-up of a prospective randomized trial. Arthroscopy. 2016;32(1):97-109. doi:10.1016/j.arthro.2015.09.010
30. Matas J, Orrego M, Amenabar D, et al. Umbilical cord-derived mesenchymal stromal cells (MSCs) for knee osteoarthritis: repeated MSC dosing is superior to a single MSC dose and to hyaluronic acid in a controlled randomized phase I/II Trial. Stem Cells Transl Med. 2019;8(3):215-224. doi:10.1002/sctm.18-0053

31. Khalifeh Soltani S, Forogh B, Ahmadbeigi N, et al. safety and efficacy of allogenic placental mesenchymal stem cells for treating knee osteoarthritis: a pilot study. Cytotherapy. 2019;21(1):54-63. doi:10.1016/j.jcyt.2018.11.003

32. Alonso-Goulart V, Ferreira LB, Duarte CA, et al. Mesenchymal stem cells from human adipose tissue and bone repair: a literature review. Biotech Res \& Inno. 2018;2(1):74-80. doi:10.1016/j.biori.2017. 10.005

33. Bowman S, Awad ME, Hamrick MW, Hunter M, Fulzele S. Recent advances in hyaluronic acid-based therapy for osteoarthritis. Clin Transl Med. 2018;7(1):6. doi:10.1186/s40169-017-0180-3

34. Champlin R Selection of Autologous or Allogeneic Transplantation. In: Kufe DW, Pollock RE, Weichselbaum RR, et al., editors. HollandFrei Cancer Medicine. 6th edition. Hamilton (ON): BC Decker; 2003. Available from: https://www.ncbi.nlm.nih.gov/books/ NBK12844/. Accessed November 26, 2020.

35. Liberati A, Altman DG, Tetzlaff J, et al. The PRISMA statement for reporting systematic reviews and meta-analyses of studies that evaluate healthcare interventions: explanation and elaboration. $B M J$. 2009;339:b2700. doi:10.1136/bmj.b2700
Stem Cells and Cloning: Advances and Applications

\section{Publish your work in this journal}

Stem Cells and Cloning: Advances and Applications is an international, peer-reviewed, open access journal. Areas of interest in established and emerging concepts in stem cell research include: Embryonic cell stems; Adult stem cells; Blastocysts; Cordblood stem cells; Stem cell transformation and culture; Therapeutic cloning; Umbilical cord blood and bone marrow cells; Laboratory,
Dovepress

animal and human therapeutic studies; Philosophical and ethical issues related to stem cell research. This journal is indexed on CAS. The manuscript management system is completely online and includes a very quick and fair peer-review system, which is al easy to use. Visit http://www.dovepress.com/testimonials.php to read real quotes from published authors. 